\title{
TRANSACCIONES COMERCIALES APLAZADAS EN LA CIUDAD DE MURCIA (1500-1515)
}

Por

CARMEN M. CREMADES GRIÑAN 


\section{INTRODUCCION}

La elaboración de este trabajo surgió ante la lectura de unas palabras con que Torres Fontes expresaba su deseo de conocer cómo se desenvolvían las formas de trabajo de los mercaderes, "sus relaciones ciudadanas y la enumeración nominal de los que traficaron con varia fortuna" (1). Por ello el análisis de las escrituras de obligación contenidas en los protocolos del Archivo Histórico de Murcia (2), aportó suficientes datos para desarrollar el principal comercio existente en la ciudad de Murcia a principios del siglo XVI. Se han seleccionado estas cartas de obligación siguiendo una trayectoria comercial, basada tan sólo en el tráfico de bienes muebles, (como paños, trigo, ganado, etc.) y también se considera la figura del esclavo, pues, con éste se comercia como si de un objeto se tratase. Por ello,

(1) "Genoveses en Murcia (siglo XV)". Miscelánea Medieval Murciana, II, (1976), pág. 71. En este interesante estudio expone sus fines, los cuales no son los de hacer historia del comercio, ni del desarrollo de la industria castellana. A la vez atrae la atención para que este proceso histórico no se rompa, sino que se prosiga en la tarea para llegar a un conocimiento más completo.

(2) La documentación existente en este Archivo (A. H. Mu.) relativa a los comienzos del siglo XVI se concreta en los protocolos $n^{\circ} 65,288,362,363,364,603$ y 608 Todos ellos han servido de fuente para el presente estudio. Tornel Cobacho, C.: Documentos notariales murcianos: 1450-1515. Tesis de licenciatura inédita. Murcia. 1979. 
quedan descartadas todas las ventas referentes a tierras, bienes inmuebles que quedan agrupadas para un posterior estudio concerniente a la propiedad agrícola.

Murcia en el contexto político de principios del siglo XVI.

Bajo los Reyes Católicos el orden imperante y los desvelos del buen gobierno alentarían la economía del reino, antes ya de que lo enriquecieran las tierras de Granada (3) y se estableciese un comercio intenso, basado en la seda, aceite y trigo. Por tanto, como repercusión se aceleró la actividad productiva, lo que favoreció una fase propicia de rendimientos. La paz aumentó el comercio y desarrolló la actividad industrial en Murcia, tras la grave epidemia de peste que asoló el territorio entre 1488 y 1489 (4), especializándose en tejidos (5). Todo ello repercutió en el cambio de fisonomía de las ciudades (6), favorecidas por las edificaciones monumentales que surgen; nuevo estilo arquitectónico, inspirado en las construcciones italianas, que exigían nuevos artífices y nuevos modos de vida: de tipo social, religioso, más abierto a la calle (7). El ajuar personal y de la casa, junto con el mobiliario de las grandes casas fomentaron las artes menores, como se observa en el estudio de los testamentos del siglo XVI (8).

Estas ideas básicas permitieron que “a una industria pobre de carácter

(3) CaRANDE. Ramón: La ecunomía y la expansión ultramarina bajo el gobierno de los Reyes Católicos, en 7 estudios de Historia de España, Barcelona, 1969, pág. 16.

(4) La población de Murcia para 1488 se cifraba en 1.750 vecinos, de los cuales murieron unas 6.088 personas. Posteriormente se recuperó demográficamente la ciudad y en 1517 se calculaban alrededor de 3.000 vecinos. (Torres Fontes. J.: "Estampas de la vida en Murcia en el reinado de los Reyes Católicos". Murgetana, 15, (1961), pág. 76).

(5) ToRRes Fontes. J : "Genoveses...", op. cit. pág. 78.

(6) TORRES FONTES, J.: "Estampas...", op. cit. pág. 55.

(7) Carande, R.: op. cit, pág. 16.

(8) Coria Colino, J.: "El testamento como fuente de estudios sobre mentalidades", Miscelánea Medieval Murciana, IX; (1982). pág. 193. 
puramente local, sin más horizonte que su propio término concejil y que importaba tejidos y paños de mediana y superior calidad, va a suceder una industria próspera que repercutirá en la vida ciudadana" (9). Esta se hallaba integrada por un nutrido sector terciario que suponía un 30 por ciento de la población total, lo que proporcionaba un consumo elevado, que favorecía notablemente el desarrollo industrial en sus diferentes ramos de la seda, paño, calzado, principalmente, "pero faltaba la burguesía que hubiese invertido el capital que generaba la venta de la tierra. Clase que era reemplazada por los mercaderes que extraían del campesinado la materia prima para destinarla a la exportación” (10).

Murcia, además, ofrecía una óptima situación geográfica al ser una encrucijada de caminos, lo que favoreció un intenso comercio, tanto terrestre, como marítimo (11). Este último se desarrollaba por el puerto de Cartagena, por "donde se enviaban productos tradicionales como higos, aceite, miel, cera, arroz, lino, azogue, bermellón, cominos, greda, lana, cueros y alumbre" (12). Todo este período político indica una fuerte tonalidad en el resurgimiento de la vida económica, "un florecimiento comercial que sale de sus límites y traspasa las fronteras del reino por contados pasos; mercaderes burgaleses sustituyen a los italianos en Murcia y murcianos son los que exportan por el Mediterráneo los productos de su Huerta y su industria." (12).

(9) Torres Fontes, J.: "Genoveses...”, op. cit., pág. 104.

(10) Chacon Jimenez, F.: Murcia en la centuria del Quinientos, Murcia, 1979, pág. 371 .

(11) Las rutas de caminos que enlazaba Murcia con otras ciudades: Valencia, Alicante, Aibacete, Granada, Aranjuez, entre otras, vienen detalladas en numerosas obras del siglo XIX. Citamos como ejemplo a LABORDE, A.: Itinerario descriptivo de las provincias de España, Valencia, 1826.

De una manera más especializada y referido al comercio y las ferias, cfr. Gual, José Miguel: "Bases para el estudio de las ferias murcianas en la Edad Media". Miscelánea Medieval Murciana, IX, (1982), págs. 9-57 (especialmente pág. 27); Chacon Jiminez, F.: La vida económica, en Historia de la Región Murciana, tomo V. Murcia, 1980, pág. 198.

(12) Torres Fontes, J.: “Genoveses...”, op. cit., pág. 74.

(13) Torres Fontes, J.: "Estampas...", op. cit., 13, (1960), pág. 42. 


\section{Los vecinos dedicados al comercio}

Los mercaderes negociaban con el tráfico de diversos géneros procedentes del propio reino de Murcia (ganaclo, lana, trigo), o bien, de lugares más lejanos, como el paño traído de Valencia, Londres o de Bretaña. No era frecuente el comerciante especializado en una sola especie (14), sino que trafica con diversos productos. Por ello es fácil que una misma persona venda paños, trigo, cueros, etc. A la vez el mercader era un excelente negociante y obtenía pinguies beneficios que luego los revierte en la concesión de préstamos (15). Esta satisfacción económica estaría en función de la mayor o menor venta y a la vez de la oscilación de precios, pues, no se observa un precio estable sobre todo en la primera década del siglo XVI, a pesar de la vigilancia del Concejo para luchar contra la regatonería, "uno de los males más peligrosos y endémicos de la vida económica de las ciudades" (16).

Junto a la figura del mercader se afianza la del corredor de comercio que aparece como tal figura durante el reinado de Alfonso XI (17). Su misión era la de mediar en los contratots y vender los bienes que se le encomendaran. Podían ser en un principio judíos o cristianos, tras la expulsión de los primeros son frecuentes las referencias a los corredores, cristianos nuevos. Habían de ser escogidos por los jurados y tener fiadores; eran renovados anualmente, como otros muchos oficios municipales en las elecciones de San Juan (18).

En el trato comercial, es decir, en el "juego" de la oferta y de la de-

(14) Excepto los marchantes de ganado que tan sólo se dedican a ello.

(15) Molina Molina, A. L.: "Mercaderes genoveses en Murcia durante la época de los Reyes Católicos (1475-1516)", Miscelánea Medieval Murciana, II, (1976), pág. 237.

(16) Chacon Jrmenez, F.: "Una contribución al estudio de las economias municipales en Castilla", Miscelónea Medieval Murciana, III, (1972), pág. 223.

(17) TORRES FonTES, J.: "Los corredores del comercio murciano en el reinado de Alfonso XI", Miscelánea Medieval Murciana, IV, (1978), pág. 237.

(18) Alonso Navarro, S.: Historias del comercio murciano, Murcia, 1978. pág. 39 . 
manda, se observaba un acercamiento del medio rural, vendedores, al urbano, compradores (19). En este comercio se ha querido subrayar de un modo especial la importancia de los mercaderes genoveses, que si bien es cierto hasta el siglo XVI, no hay que oscurecer otras figuras de comerciantes murcianos, como lo fueron Ginés de Cartagena, Salvador Navarro (20), Pedro Ruiz, Luis Madrid, Luis Moreno y otros tantos que acapararon el comercio a partir de 1506.

La presencia de los primeros se remonta al siglo XIII y se encuentra documentada en privilegio dado por Fernando III, datado de 1251 (21). Torres Fontes subraya la presencia de los mercaderes pisanos, genoveses y sicilianos en el texto de las Cantigas (22). La importancia de estos mercaderes ha sido estudiada por Carande (23), Torres Fontes (24), Ruth Pike (25), Molina Molina (26) y Franco Silva (27). Una vez establecidos en

(19) Torres Fontes, J.: "Los corrdores...", op. cit., pág. 240.

(20) Vecino de Murcia, el cual concentro en sus manos gran parte del comercio murciano. Se convirtió en el mayor prestamista, sobresaliendo en estas actuaciones a los genoveses que tan sólo se centró en el comercio de joyas y de chamelote desde comienzos del siglo XVI. Esta situación tan relevante se puso de manifiesto al serle concedido en arrendamiento diversos beneficios por Martín de Selva, canónigo de la iglesia de Cartagena, vecino de Murcia. El contenido de la escritura de arriendo estaba formado: 1. Los beneficios que el citado Martín de Selva tiene en las iglesias de San Clemente y San Pedro de Lorca, por precio de $12.000 \mathrm{mrs}$; 2. Un préstamo que tenía el arrendador en la villa de Mula por precio de $12.000 \mathrm{mrs} . ; 3 .^{\circ}$ un beneficio que disfrutaba en la villa de Tobarra por precio de 27 ducados; $4 .^{\circ}$ Un beneflcio y retoria de Sax por precio de 25.000 mrs.; 5." Un préstamo que tenía en Alcalá del Río por precio de 25 ducados; $6 .^{\circ}$ Los graneros y calongias de las ciudades de Murcia, Lorca y Orihuela por precio de $18.000 \mathrm{mrs}$. Todo lo citado se arrendó por tiempo de dos años. (A.H.Mu. Protocolo n. ${ }^{\circ}$ 603, fols. $13 r^{\circ}-14 r^{0}$; 1510-II-15, carta de arrendamiento).

(21) Torres Fontes, J.: "Genoveses...", op. cit., pág. 73.

(22) Torres Fontes, J.: "Los corredores...", op. cit., pág. 241.

(23) Sevilla, Fortaleza y Mercado. Universidad de Sevilla, 1972, págs. 70-81.

(24) "Genoveses en Murcia (siglo XV)", Miscelánea Medieval Murciana, II. (1976), págs 69-169.

(25) Enterprise and adventure. The Genovese in Seville and the opning of the New World, Itaca, New York, 1696.

(26) "Mercaderes genoveses en Murcia durante la época de los Reyes Católicos (1475-1516)",Miscelánea Medieval Murciana, II, (1976), págs. 277-312; La vida de la ciudad de Murcia en la Baja Edad Media, discurso leído en el ingreso como numerario de la Academia de Alfonso X el Sabio, Murcia, 1983.

(27) "El alumbre murciano", Miscelánea Medieval Murciana, VI, (1980), páginas 237-273. 
la ciudad se les ofrecía la vecindad con toda clase de facilidades (28). Esta otracción por los nuevos habitantes comenzó con Enrique II y adquirió mayor importancia con la disposición de Juan I, dada en 1384 al otorgar franquicias y exenciones a los que venían a morar en sus reinos por un tiempo superior a 10 años (29). La vecindad se solicitaba por escrito, especificando una cantidad que estaba en relación a su stattus social. Para la concesión era necesario que fuesen representados por dos vecinos y garantizasen con sus personas y sus bienes el cumplimiento de sus deberes de vecindad (29). La identificación con el patriciado urbano era favorable en numerosos casos (30), aunque también existieron envidias y recelos. Tanto para estos mercaderes afincados en las tierras del Segura, como para los oriundos del lugar fueron dadas el 5 de junio de 1470 unas disposiciones en las que se expresaban los derechos y deberes que les concernían (31).

La compra de los diversos géneros existentes en el mercado podía ser pagada de inmediato o realizarla a través de una obligación efectuada ante un escribano. En ella se establece una fecha muy variable, que puede ser próxima a la venta o lejana, esta modalidad era frecuente cuando la transacción importaba una elevada cuantía. El impago de lo prometido y obligado estaba sancionado con una stipulatio poenae, o cláusula penal que sin excepción, cra el doble de la cantidad debida.

Las diversas transacciones económicas.

Teniendo como base las fuentes documentales, ya comentadas, concernientes a los quince primeros años, se han elaborado una serie de tablas, clasificadas según las variantes repetidas registradas, las cuales permiten

(28) Tonres Fontes, J.: "Los genoveses", op. cit., pág. 95.

(29) Al ser un tiempo inferior se les menciona como habitantes de Murcia (A.H.Mu. Protocolo n. 382 , fol. 507).

(29) Torres Fontes, J.: "Genoveses...". op. cit., pág. 96.

(30) Molina Molina, A. L.: "Mercaderes...", op. cit., pág. 285.

(31) Torres Fontes, J.: "Genoveses...", op. cit., pág. 95, Apéndice n." 17. 
una visión en conjunto rápida y concisa de las principales constantes reflejadas en las escrituras de obligación.

El comentario que se realiza a las mismas se ha pretendido que fuese lo más equilibrado posible, pero el referente al comercio del paño merece un apartado especial, puesto que su importancia es muy elevada frente a los otros tratos comerciales.

\section{COMERCIO CON EL PANO}

Las diversas variantes de este género son detalladas en las diversas escrituras así, pues, tenemos. De una calidad inferior, pero muy frecuente es la del paño dieciocheno de la ciudad que se vendía en diversas tonalidades: negro, rojo, verde y rosado. Su procedencia podía ser la de la propia ciudad o de otras, como de Cuenca o de Valencia. De origen extranjero se menciona el de Brujas. El precio de esta calidad era muy variable desde 200 mrs. a $230 \mathrm{mrs}$, en 1510 su valor disminuye a $136 \mathrm{mrs}$. La calidad podía ser fina, brujulada y buriel, influyendo la tonalidad.

Una segunda clase de tejido de superior textura era la constituida por el veinteno o veintedoseno, de diferentes y alegres colores: verde, morado, plateado, rosado, verde alimonado y negro. Su procedencia se centraba en Murcia y en Valencia. Su precio era algo más elevado que el tipo anteriormente citado, oscilaba entre los 340 a los 260 mrs. En 1510 se llegó a vender la vara a $575 \mathrm{mrs}$.

Otras clases de tejidos de mejor calidad lo componían los de contrai, el fustán, ruan y palmilla entre otros. Los paños del primer tipo procedían de Valencia y se vendían a pocos comerciantes en cantidades elevadas a un precio aproximado de unos $750 \mathrm{mrs}$. en 1510. La cotización más alta la alcanzaba el paño de grano a $800 \mathrm{mrs}$. la vara. 
Los compradores eran vecinos de Murcia, principalmente, aunque también de Alcantarilla, Librilla, Alhama, Molina, Nora, Jumilla y Caravaca. Sus oficios eran varios, y muy insuficientemente se citan, entre otros: labradores, zapateros, cantareros, hortelanos y entre las mujeres, muy escasas, destacan las viudas. A menudo el sexo débil era representado por otros individuos.

Por otro lado, los comerciantes muy diversos a principios del siglo XVI, en 1510 se concentran en unos pocos mercaderes y entre ellos señalamos a Ginés de Cartagena, Salvador Navarro y Francisco Ruiz, principalmente. Todos ellos eran vecinos de Murcia; eran genoveses Diego de Zamora, Domenego Maza, Juancho Oñate, Francisco Jufre, Cofia y Rey. Algunos citados como vecinos y otros como habitantes de Murcia. Los oficios de estos vendedores eran diversos, si bien algunos de ellos eran citados como mercaderes, como todos los genoveses y algunos otros, son normalmente en la venta de paños traperos, como Francisco Ruiz, boticarios, perailes e incluso notarios, sobresaliendo Bartolomé Coque.

La venta más numerosa tenía lugar en los meses de marzo y de diciembre. Los pagos aplazados se fijaban preferentemente en San Juan de junio y en la Pascua de Resurrección.

La unidad principal de medida de longitud era la vara, equivalente aproximadamente a $835 \mathrm{~mm}$. y 9 décimas. Unidades inferiores se denominaban como las fracciones, sólo en algunos casos se cita la sesma.

El pago se efectuaba en maravedises hasta 1507, a partir de entonces se entregaban ducados de oro (32) y reales de plata. En algunos casos reducidos se efectuaba un trueque entre dos especies, como se advierte en la transacción efectuada entre Bartolomé Albacete, peraile, vecino de Mur-

(32) Esta moneda acuñada desde el siglo XIII, se reguló por la pragmática de Medina del Campo, dada el 3 de junio de 1497, durante los dos primeros siglos de la Edad Moderna adquirió gran apogeo, no obstante en las transacciones pequeñas predominó el maravedí (Carande, R.: op. cit., pág. 30). 
cia que vendió a Fernando Benito una vara de paño veinteno negro a cambio de la entrega de 3 fanegas de trigo. Intercambio que equivalía a 1.200 mrs.

Es preciso subrayar que las nuevas técnicas y la abundancia, diversidad y mejor calidad de los tintes, permiten un desarrollo continuado en la confección y tintado de los paños y lanas de todas clases (33). La especialidad de tinturas la monopolizaban los comerciantes extranjeros desde la Baja Edad Media (34).

Comercio con la lana.

Muy reducido debido a la compra de paños extranjeros (35), según se puede apreciar en las escrituras de obligación, lo que supone poco comercio en esta ciudad sobre este género o por lo que se refiere a pagos aplazados. Afirmación que es difícil de mantener si se establece un paralelismo con las transacciones efectuadas con el paño; la regularidad de precios no era frecuente, pues, lo mismo se vendía la arroba a 525 mrs., en 1506 , como posteriormente disminuye a $300 \mathrm{mrs}$. en 1510. Los vendedores eran vecinos de Murcia, Albacete, y de Caravaca; sus oficios se desconocen. Sin embargo los compradores eran guanteros, entre ellos sobresale maestre Alonso y un genovés Felipe Escaja (35).

Los pagos aplazados no obedecen a un ritmo fijado, sino más bien a la entrega en dos veces de la cantidad estipulada, los períodos podían ser desde Carnestolendas hasta S. Juan. Como peculiaridades podemos citar el pago de 130 arrobas compradas por Jaime Mariner, vecino de Caravaca a Francisco Maynete, vecino de Murcia, con 52 varas de terciopelo negro, valorado en $760 \mathrm{mrs}$. la vara. Tal adquisición se efectuó el 4 de

(33) Torres Fontes, J.: "Genoveses...", op. cit., pág. 104.

(34) Torres Fontes, J.: “Estampas...”, op. cit., 16, (1961), págs. 39 y 44.

Cfr. para la evolución de todo el engranage comercial de paños las Tablas números I-XIII.

(35) Torres Fontes, J.: "Estampas...", op. cit., pág. 58. 
julio de 1510, obligándose el comprador a entregar la mencionada cantidad en enero del año siguiente (37).

Comercio de ganado.

Las compras efectuadas se remiten principalmente a una unidad, como potros, mulas, machos cerriles, asnos, bueyes, caballos y yeguas, entre otros. En cuanto a ganado ovino (38) no existe ninguna mención sobre transacciones, excepto la que se refiere a un trueque habido entre un rebaño de ovejas y otro de borregos. Este curioso intercambio se realizó por Alonso Fernández, vecino de Mula, y Mateo Fernández, vecino de Moratalla. El primero compró 500 ovejas, valoradas en $130 \mathrm{mrs}$. cada una, comprometiéndose a entregar 250 borregos durante cuatro años, valorada cada unidad en $65 \mathrm{mrs}$.

Los compradores proceden de Mula, Cartagena, Puebla de Soto, Requena y de la misma ciudad. Los vendedores vienen de Moratalla, Puebla de Soto, Murcia y de Hellin. Siendo de este pueblo Fernando Jiménez, principal marchante de ganadería. Los oficios de estos últimos son diversos, como se puede apreciar en las tablas números XV-XVII: notario, tintorero, racionero de la Iglesia de Cartagena, escribano del tribunal de la Santa Inquisición, aserrador, etc.

(36) Compró el 16 de enero de 1507 a Andrés Carrasco de Caravaca toda la lana blanca y fina que se obtuviese de su ganado, valorada a $420 \mathrm{mrs}$. la arroba. Su pago se realizaria al esquilrse la lana, entregando la mitad de su valor y el resto en julio de 1508 .

(37) Cfr. la Tabla n. XIV.

(38) El descontento por la compra de paños extranjeros se puso de manifiesto en las cartas del Concejo dirigidas a los Reyes Católicos, los cuales se hicieron "eco de la solicitud murciana de que se prohibiera la entrada de paños extranjeros ya que esta importación habia ocasionado entre otros males, el que "los que tenian ganado lo vendiesen, de manera que de cincunta mill ovejas que habia en la dicha cibdad, non quedaron ocho o diez mill". (TorRes Fontes, J.: "Estampas...", op. cit. 15. (1961), pág. 88. 
Comercio de la seda.

Esta industria quedó gravemente mermada por la expulsión de los judíos (39), y de esta crisis se recuperaría lentamente en los siglos XVI y XVII. La venta de la seda, tanto elaborada, como en bruto, era poco frecuente en el sistema de pagos aplazados, tal como se refleja en las escrituras de protocolos examinados. Las cantidades eran pequeñas, de una a dos varas, y la calidad comprada era la de seda rasa negra, principalmente. Cuando se vendía en bruto, se entregaba en unidades de peso como la libra. El precio oscilaba entre los 400 y $450 \mathrm{mrs}$., tanto para la fabricada, como para el segundo tipo.

Relacionada con este género, detallamos la compra de la hoja de morera para alimentar los gusanos de la seda. El cómputo se realizaba, no por peso, sino por la producción total de las moreras, quedando enumeradas las que se adquirían. La compra se realizaba a principios del año, comprometiendo la hoja que hubiese para la primavera próxima, aunque existen obligaciones por la producción de dos años consecutivos. El precio por morera varía desde 75 mrs. a los 95 . La compra de la hoja se realiza directamente al pequeño, arrendador, enfiteuta, o mediero que venden su producto a buen precio. Este hecho potenciaría la renta de la tierra (40). Tanto los compradores como los vendedores eran vecinos de Murcia (41).

Comercio del trigo.

Este comercio realizado con anterioridad a la creación del Pósito, permite establecer una relación de comprador-vendedor, que si bien no se de-

(39) Torres Fontes, J.: "Estampas...", op. cit,, 11, (1958), pág. 31.

(40) Chacon Jimenez, F.: Murcia en la centuria del Quinientos, op. cit., pág. 342. Menciona los precios medios por cada onza de hoja.

(41) Cer. las Tablas n.* XVIII-XIX. 
talla su profesión debemos pensar que gran parte de ellos eran panaderos, molineros, o bien particulares, propietarios de un horno, como sucede con Diego Zambrana, Alonso de Tenza, y Juana Manuel, todos ellos vecinos de la Puebla de Soto (42). Las cantidades no superaban los dos cahices, unas 24 fanegas, valorados según la pragmática. Este precio era normalmente respetado, y se situaba entre los 400 mrs. hasta 1510 que subió a los $550 \mathrm{mrs}$. el cahiz, es decir unos $45 \mathrm{mrs}$. la fanega. También en este año citado se presentaría una novedad en lo que concierne a los vendedores: si bien a comienzos de siglo son varios, a partir de la segunda década del quinientos la venta la dirigen dos marchantes que sobresalen en el comercio de otros géneros, nos referimos a Salvador Navarro y a Pedro Ruiz, ambos vecinos de Murcia. El plazo establecido para la entrega del trigo era el de San Juan de junio.

El trigo se cultivaba de un modo intenso en la Huerta y en el campo se procedía de un modo de rotación bienal (43). La crisis de comienzos de siglo fue notable y se acusó, especialmente entre los años de 1502 a 1509 (44). Este factor favoreció el intercambio de grano por otros géneros de diversa índole. También se observa el pago del arrendamiento de animales en cantidades de trigo; a la vez era frecuente la entrega de una cierta cantidad de cebada como pago de la compra de unas tierras, como también la satisfacción de los réditos censuales (45).

Comercio de varios géneros

Frente a lo que pudiese parecer, el comercio aplazado de géneros diversos no era abundante. Se comercializaba con diferentes productos, co-

(42) Cremades Griñan, C. M.a: "Connotaciones a los censos del Santo Oficio (1535-1600) en la ciudad dee Murcia". Murgetana (en prensa).

(43) Tornel Cobacho, C.: "El problema del trigo en Murcia en la época de los Reyes Católicos", Miscelánea Medieval Murciana, V, (1980), pág. 60.

(44) Ibidem, pág. 67; Vicens VIVEs, J.: La economia de los Reyes Católicus, en Historia Económica de España, Barcelona, 1959, pág. 277.

(45) Cfr. las Tablas n." XX-XXI-XXII. 
mo cueros, joyas, papel, pimienta, queso, miel (46), almendras, baldosas, tintes, sardinas, etc. Debemos señalar la preponderancia de los mercaderes genoveses en este sector, sobre todo en lo que se refiere a las joyas: Francisco Espinardo, Antonio Grimaldo, Tomás de Guiso, Jacomo Cofia, principalmente.

El pago del plazo es variado: San Juan, Pascua Florida, San Miguel, Navidad, etc. Se registran diversas unidades en la venta de los géneros: libras, arrobas, piezas - piezas en lo que se refiere a paños- varas, balas - para el papel-, sarrias en la venta de las sardinas. Como original destacamos la venta de los guantes en la cual se establece como precio unidad la veintena.

Reflexiones sobre las escrituras de obligaciones.

En torno a la variante suscitada en este estudio se observa la poca dinamicidad existente en el modo de pago aplazado, este fenómeno se puede explicar bien por la situación acomodada de la sociedad murciana, o porque este tipo de satisfacer las compras efectuadas no fuese habitual.

De ahí observamos que escrituras de paños existen unas 204, lo que indica comercio muy frecuente $y$ numeroso frente a otros géneros. Les siguen en menor cantidad las referidas a áridos, unas 50, y las de compra de ganado, aproximadamente unos 42; de menor importancia son las referentes a seda y lana.

Los datos reflejados en este estudio son una muestra del desarrollo del tráfico mercantil en los primeros tiempos renacentistas, de esa actividad incesante y oscura que teje gran parte de la trama de las relaciones interpersonales en una comunidad. Con frecuencia el historiador se des-

(46) La miel y la cera eran las bases de una intensa explotación comercial, herencia de la economía musulmana, mantenida en el transcurso de los siglos medievales y con pervivencia de la Edad Moderna. (Torres Fontes, J.: "Estampas...", op. cit., 24 (1965), pág. 46). 
lumbra y, ello es positivo, ante el fulgor de los grandes acontecimientos que marcan cumbres en el devenir histórico. Pero por debajo de los hechos sobresalientes discurre un continuo hormiguero de hombres que sobreviven solucionando los minúsculos problemas cotidianos, satisfaciendo sus necesidades perentorias, procurándose el yantar, el vestido, la fuerza de trabajo para labrar su tierra o el pequeño lujo que les permita sentirse importantes. La comprensión de la Historia pasa tanto por el estudio del esforzado guerrero y el hábil político, como por el trajinero que provee a los vecinos de los productos necesarios para la subsistencia. $Y$ no hay que desdeñar el influjo que a la larga tendrán estas pequeñas circunstancias cotidianas sobre la producción de los grandes hechos sociales, porque la práctica del tráfico diario va a crear o modificar los planteamientos económicos, la estratificación social $\mathrm{y}$, sobre todo, la mentalidad de los in. dividuos.

El desarrollo del comercio como actividad de intermediación llevada a cabo por profesionales, encuentra sus raices en la Edad Antigua. La actividad comercial rompe los esquemas de una economía cerrada y feudal, favoreciendo la cultura ciudadana de la baja Edad Media. Pero desde los albores de la Edad Moderna, que es el tiempo objeto de este estudio, se afianza y se potencia como uno de los soportes de los nuevos estados nacionales. De forma lenta, casi insensible, la sociedad se va haciendo más dependiente de los comerciantes, sin los cuales no puede apenas asegurar la subsistencia, como se observa al interrumpirse las comunicaciones en caso de inundaciones; y la clase social de la burguesía, en gran parte comerciante, irá ascendiendo a expensas de la nobleza. Esta burguesía cada día más potente supondrá uno de los soportes que apuntalen el Antiguo Régimen, pero también será en gran medida el detonante que lo haga derrumbarse. 
VENTAS DE DISTINTOS GENEROS DE PAÑOS - TABLA N. I (1505)

\begin{tabular}{|c|c|c|c|c|c|c|c|c|c|c|}
\hline \multicolumn{3}{|c|}{ COMPRADOR } & \multicolumn{3}{|c|}{ VENDEDOR } & \multirow[b]{2}{*}{ Prrcio } & \multirow[b]{2}{*}{ Cantidad } & \multirow[b]{2}{*}{ Esperis } & \multirow[b]{2}{*}{ Plasos } & \multirow[b]{2}{*}{ PROTOCOLO } \\
\hline Tirular & Oficio & Verino & Tizular & Ofirio & Vecino & & & & & \\
\hline Fndo. Carreño & & Murcia & Gines Cartagena & mercader & Murcia & 200 mrs. & I vara y sesma & $\begin{array}{l}\text { dieciocheno } \\
\text { ciudad }\end{array}$ & Pascua & 362-F. 309-310 \\
\hline Andres Morales & & Murcia & Francisco Ruiz & trapero & Murcia & 220 mrs. & 1 vara & $\begin{array}{l}\text { dieciocheno } \\
\text { verde os. }\end{array}$ & Pascua & 362-F. 310-311 \\
\hline Ginés Leơn & & Murcia & Juancho Onate & & Murcia & $910 \mathrm{mrs}$. & 3 varas y cuarta & $\begin{array}{l}\text { veinteno } \\
\text { plateado }\end{array}$ & Pascua & 608-F. $89-90$ \\
\hline Juan Fernández & & Murcia & Juan Garre & & Murcia & $1.050 \mathrm{mrs}$ & 5 var2s & $\begin{array}{l}\text { dieciocheno } \\
\text { fino }\end{array}$ & $\begin{array}{l}\text { Sta. M: } \\
\text { agosto }\end{array}$ & $608 \cdot F \cdot 94-95$ \\
\hline Pedro Guerán & & Murcia & Andrés Fernández & & Murcia & $400 \mathrm{mrs}$. & 2 varas y media & burdicl & S. Juan & 608-F.95-96 \\
\hline Pedro Guerán & & Murcia & Bartolomé Coque & notario & Murcia & 1.155 mrs. & 5 varas y media & dieciocheno & S. Mfiguel & $608-\mathrm{F} .96 \mathrm{r}^{\circ}-\mathrm{v}^{\circ}$ \\
\hline Juan de Jumilla & & Murcia & Francisco Gómez & & Murcia & $1360 \mathrm{mrs}$ & 4 varas & veintedoseno & S. Juan & 608-F. 98-99 \\
\hline Luis Carrillo & & Murcia & Ginés Roldán & & Murcia & $840 \mathrm{mrs}$. & 7 varas & buricl & S. Juan & $608-F .100-101$. \\
\hline Catalina Ruiz & Vda. & Murcia & Francisco Garcia & & Murcia & $918 \mathrm{mrs}$. & 4 varas y media & $\begin{array}{l}\text { dieciocheno } \\
\text { negro }\end{array}$ & S. Juan & $608-F .103 v^{\circ}$ \\
\hline Alonso Gómez & & Murcia & Juan Rivas & & Murcia & 1.575 mrs. & 7 varas y media & $\begin{array}{l}\text { dieciocheno } \\
\text { negro }\end{array}$ & S. Juan & 608-F. 107-108 \\
\hline M. Sánchez & & Murcia & Salvador Navarro & mercader & Murcia & $800 \mathrm{mrs}$. & 20 varas & Bretaña & $\begin{array}{l}\text { Sta M.: } \\
\text { septiembre }\end{array}$ & 608-F.108-109 \\
\hline Juan Tomís & labrador & Murcia & Juan Garcia de P. & & Murcia & $900 \mathrm{mrs}$. & 5 varas & $\begin{array}{l}\text { dieciocheno } \\
\text { enrojado }\end{array}$ & S. Juan & 608-F. $111 \mathrm{v}^{\circ}$ \\
\hline Espuche & & Murcia & Juan Rivas & & Murcia & $1.150 \mathrm{mrs}$. & 3 varas y media & contrai & S. Juan & $608-F .114-115 r^{\circ}$ \\
\hline Alonso Martinez & & Murcia & Juan Garcia de P. & & Murcia & $510 \mathrm{mrs}$. & 3 varas & enrojado & S. Juan & $608-F .117-118 r^{\circ}$ \\
\hline Pedro Garcla & cantarero & Murcia & Ginés Roldán & & Murcia & $612 \mathrm{mrs}$. & 4 varas y media & enrojado & Pascua & $608-\mathrm{F}, 118 \mathrm{r}^{\circ}, v^{\circ}$ \\
\hline Findo. Jumilla & albanil & Murcia & Salvador Navarro & mercader & Murcia & $6.750 \mathrm{mrs}$. & 30 varas & Bretaña & Pascua & $608-F, 122 v^{\circ}-123$ \\
\hline Alonso Garefa & trajinero & Murcia & Francisco Gómez & & Murcia & $475 \mathrm{mrs}$. & 3 varas y media & veinteno & S. Juan & 608-F. 123v' \\
\hline
\end{tabular}


VENTAS DE DISTINTOS GENEROS DE PAÑOS - TABLA N. II (1506)

\begin{tabular}{|c|c|c|c|c|c|c|c|c|c|c|}
\hline \multicolumn{3}{|c|}{ COMPRADOR } & \multicolumn{3}{|c|}{ VENDEDOR } & \multirow[b]{2}{*}{ Precio } & \multirow[b]{2}{*}{ Cantidad } & \multirow[b]{2}{*}{ Especic } & \multirow[b]{2}{*}{ Plazo } & \multirow[b]{2}{*}{ PROTOCOLO } \\
\hline Titular & Oficio & Vecino & Titslar & Oficio & Vecino & & & & & \\
\hline Francisco de Siles & labrador & Murcia & Juan Roldán & & Murcia & $646 \mathrm{mrs}$. & 5 varas $-1 / 4$ & buriel & Santa M. & 608-F. 123-124 \\
\hline Catalina Garça & Vda. & Murcia & Juan Rivas & & Murcia & $2.000 \mathrm{mrs}$ & & & S. Juan & 608-F. 126r, $v^{*}$ \\
\hline Martín Morata & & Murcia & Pedro de Monreal & & Murcia & $863 \mathrm{mrs}$ & 2 varas y media & & $\begin{array}{l}\text { Santa M. } \\
\text { agosto }\end{array}$ & 608-F. 130-131 \\
\hline Juan Ramirez & elérigo & Murcia & Salvador Navarro & & Murcia & $1.210 \mathrm{mrs}$ & 5 varas y media & $\begin{array}{l}\text { diociocheno } \\
\text { verde y } n .\end{array}$ & S. Juan & $608-F .132 r^{\circ}, v^{*}$ \\
\hline Andrés Forte & & Murcia & Gonzalo López & & Murcia & $675 \mathrm{mrs}$. & 2 varas y cuarta & $\begin{array}{l}\text { veinteno } \\
\text { morado }\end{array}$ & S. Juan & 608-F. $132 v^{*}-133$ \\
\hline Francisco Celdrán & albardero & Murcia & Juan de Buenavida & & Murcia & $2.420 \mathrm{mrs}$ & 110 varas & jerga & 4 meses & 608-F. $135 r^{\circ}-136$ \\
\hline Juan Dlaz & tundidor & Alhama & Salvador Navarro & mercader & Murcia & $2.175 \mathrm{mrs}$ & 7 varas y cuarto & veinteno & S. Juan & 608-F. 137r, $v^{\circ}$ \\
\hline Pedro Dacora & & Alcantarill & Salvador Navarro & mercader & Murcia & 800 mrs. & 5 varas & buriel & S. Juan & $608-F .137 v^{\circ}-138$ \\
\hline Alonso de Jorquera & & Murcia & Pedro Monreal & & Murcia & $595 \mathrm{mrs}$. & 2 varas y media & verde osc. & S. Juan & $608-F .138 v^{*}-139$ \\
\hline Luis Arroniz & cantarero & Murcia & Salvador Navarro & mercader & Murcia & $5.270 \mathrm{mrs}$ & 27 varas & $\begin{array}{l}\text { dieciocheno } \\
\text { ver. }\end{array}$ & un affo & 608-F. $145 r^{\circ}, v^{\circ}$ \\
\hline Talón el Blanco & & Ricote & Pedro Monreal & & Murcia & $1.435 \mathrm{mrs}$ & 4 varas y media & $\begin{array}{l}\text { paño rosado, } \\
\text { amarillo }\end{array}$ & S. Miguel & 608-F. 146- $\mathrm{r}^{\circ}, \mathrm{v}^{\circ}$ \\
\hline Pedro Garcla & & Murcia & Salvador Navarro & mercader & Murcia & $647 \mathrm{mrs}$. & $\begin{array}{l}2 \text { varas y cuarto } \\
1 / 2 \text { vara }\end{array}$ & $\begin{array}{l}\text { paño negro } \\
\text { veinteno }\end{array}$ & $\begin{array}{l}\text { Sta. M: } \\
\text { agosto }\end{array}$ & $608-F .147 v^{\circ}-148$ \\
\hline Francisco Cardona & & Murcia & Francisco Gómez & & Murcia & $1.400 \mathrm{mrs}$ & 7 varas & paño negro & Pascua & 608-F. 148-149 \\
\hline Bartolomé Muñoz & & Murcia & Diego Guevara & & Murcia & $900 \mathrm{mrs}$. & 5 varas & $\begin{array}{l}\text { dieciocheno } \\
\text { ros. }\end{array}$ & S. Juan & 608-F. $161 v^{*}-162$ \\
\hline Jaime Chinchilla & & Murcia & Juan Garcia Plas & & Murcia & 543 mrs. & 3 varas y media & buriel & $\begin{array}{l}\text { Sta M. } \\
\text { agosto }\end{array}$ & $608-F .162 v-163 r$ \\
\hline Pedro Tomás & labrador & Murcia & Gonzalo López & & Murcia & 700 mrs. & 3 varas y media & buriel & S. Juan & $608-F \cdot 163 r^{\circ}-164$ \\
\hline Juan Cabrero & & Murcia & Gonzalo López & & Murcia & $420 \mathrm{mrs}$ & 2 varas y media & verde oscuro & S. Juan & $608-F .164 v-165 r$ \\
\hline
\end{tabular}




\section{VENTAS DE DISTINTOS GENEROS DE PAÑOS - TABLA N.`III (1506)}

\begin{tabular}{|c|c|c|c|c|c|c|c|c|c|c|}
\hline \multirow[b]{2}{*}{ Titular } & \multirow[b]{2}{*}{ Oficio } & \multirow[b]{2}{*}{ Vecino } & \multicolumn{3}{|c|}{ VENDEDOR } & \multirow[b]{2}{*}{ Precio } & \multirow[b]{2}{*}{ Cantidad } & \multirow[b]{2}{*}{ Especie } & \multirow[b]{2}{*}{ Plasoso } & \multirow[b]{2}{*}{ PROTOCOLO } \\
\hline & & & Titular & Oficio & Vecino & & & & & \\
\hline Diego Pellicer & notario & Murcia & Juancho Oñate & mercader & Murcia & $1.212 \mathrm{mrs}$ & 2 varas y cuarto & $\begin{array}{l}\text { veintedo } \\
\text { Valencia }\end{array}$ & S. Juan & $362-F .278 r^{\circ}, v^{\circ}$ \\
\hline Isabel de Brumbell & viuda & Murcia & Alonso del Monte & trapero & Murcia & $1.100 \mathrm{mrs}$. & 5 varas y media & $\begin{array}{l}\text { diecioch. } \\
\text { Brujas }\end{array}$ & S. Juan & $362-F .278 r^{\circ}, v^{\circ}$ \\
\hline Martín Fernández & labrador & Murcia & Ginés de Cartagena & mercader & Murcia & $622 \mathrm{mrs}$ & $\begin{array}{l}2 \text { varas y } \\
1 \text { tercio }\end{array}$ & buriel & Navidad & 362-F. 279 $r^{\circ}, v^{\circ}$ \\
\hline Juan Navarro & tintorero & Murcia & Juan Nadal & & Murcia & $500 \mathrm{mrs}$ & 4 varas & $\begin{array}{l}\text { buriel die- } \\
\text { ciocheno, for. }\end{array}$ & S. Juan & 362-F. 578 $r^{\circ}, v^{\circ}$ \\
\hline Bartolomé Bellver & & Murcia & Ginés Cartagena & mercader & Murcia & $545 \mathrm{mrs}$. & 3 varas & forastero & S. Juan & $362-\mathrm{F} .530 \mathrm{r}^{\circ}$ \\
\hline Alonso Pintado & & Librilla & Gines Cartagena & 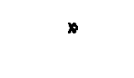 & Murcia & 884 mrs. & $\begin{array}{l}2 \text { capotes } \\
2 \text { varas y media }\end{array}$ & $\begin{array}{l}\text { sayal } \\
\text { buriel }\end{array}$ & S. Juan & $362-F .531 r^{\circ}, v^{*}$ \\
\hline Alonso Martíncz & & Murcia & Ginés Cartagena & $"$ & Murcia & & $\begin{array}{l}1 \text { manta blanca } \\
1 / 2 \text { paño morado }\end{array}$ & & S. Juan & 362-F. 532 \\
\hline Juan de Jerez & & Murcia & Gines Cartagena & " & Murcia & $510 \mathrm{mrs}$ & 1 manta blanca & & S. Juan & $362-F .540 r^{\circ}$ \\
\hline Pedro Francisco & & Murcia & Ginés Cartagena & $"$ & Murcia & $380 \mathrm{mrs}$. & 3 varas y $3 / 4$ & $\begin{array}{l}\text { paño buriel } \\
\text { forastero }\end{array}$ & S. Juan & 362-F. $541 r^{*}, v^{\circ}$ \\
\hline Miguel Mingraño & & Murcia & Gines Cartagena & $"$ & Murcia & $816 \mathrm{mrs}$. & $3 / 4$ varas & $\begin{array}{l}\text { pario blan } \\
\text { verde mor }\end{array}$ & S. Juan & $362-F .542 r^{\circ}, v^{\circ}$ \\
\hline Juan de Huete & & Alcantari & a Ginés Cartagena & $"$ & Murcia & $1.504 \mathrm{mrs}$. & 6 varas & $\begin{array}{l}\text { pafto } \\
\text { dieciocheno }\end{array}$ & S. Juan & 362-F. 544r, $v^{\circ}$ \\
\hline Fernando Antolino & & Murcia & Catalina Oller & & Murcia & $800 \mathrm{mrs}$. & 4 varas & $\begin{array}{l}\text { buriel } \\
\text { dieciocheno }\end{array}$ & $\begin{array}{l}\text { 1/2 S. Juan } \\
1 / 2 \text { Pascua }\end{array}$ & 362-F. 546r", $v^{\circ}$ \\
\hline Alonso Toledo & cantarero & Murcia & Vicencio de Guisa & genovés & Murcia & $1.000 \mathrm{mrs}$ & 1 pieza & fustán negro & 8 meses & 362-F. 548 $\mathrm{r}^{\circ}, \mathrm{v}^{\circ}$ \\
\hline Isabel Martíncz & viuda & Murcia & Ginés Cartagena & mercader & Murcia & 750 mrs. & 3 varas y media & paño buriel d. & $\begin{array}{l}1 / 2 \text { Pascua } \\
1 / 2 \text { S. Juan }\end{array}$ & 362-F. 550r", $v^{*}$ \\
\hline Ferrán Garcia & & Murcia & Ginés Cartagena & 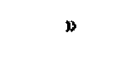 & Murcia & $650 \mathrm{mrs}$ & $\begin{array}{l}1 / 2 \text { vara morada } \\
4 \text { varas y media }\end{array}$ & $\begin{array}{l}\text { morada } \\
\text { negro }\end{array}$ & S. Juan & $362-F .550 r^{\circ}, v^{\circ}$ \\
\hline Alon. de Villanueva & a calero & Murcia & Ginés de Cartagena & w & Murcia & $1.071 \mathrm{mrs}$. & $\begin{array}{l}1 / 2 \text { vara } \\
4 \text { varas y media } \\
3 / 4 \text { vara }\end{array}$ & $\begin{array}{l}\text { morado } \\
\text { veinteno } \\
\text { albercoca. }\end{array}$ & S. Juan & $362-F .555 r^{\circ}, v^{\circ}$ \\
\hline Catalina García & & Murcia & Ginés Cartagena & $m$ & Murcia & 552 mrs. & 4 varas & paño buriel & S. Juan & 362-F. $561 r^{\circ}-562$ \\
\hline
\end{tabular}


VENTAS DE DISTINTOS GENEROS DE PAÑOS - TABLA N.' IV (1506)

\begin{tabular}{|c|c|c|c|c|c|c|c|c|c|}
\hline \multicolumn{3}{|c|}{ COMPRADOR } & \multicolumn{2}{|l|}{ VENDEDOR } & \multirow[b]{2}{*}{ Precio } & \multirow[b]{2}{*}{ Cantidad } & \multirow[b]{2}{*}{ Esperie } & \multirow[b]{2}{*}{ Plaso } & \multirow[b]{2}{*}{ PROTOCOLO } \\
\hline Titular & Ofisio & Verino & Tirular & Vecino & & & & & \\
\hline Catalina Garcin & & Murcia & Gints de Cartagena mercader & Murcia & $552 \mathrm{mrs}$. & 4 varas & buriel & S. Juan & $362-F .561 r^{\circ}, v^{\circ}$ \\
\hline Miguel Sánchez & & Murcia & Ginés de Cartagena mereader & Murcia & $850 \mathrm{mrs}$. & 5 varas & dieciocheno & S. Jixan & $362-F .562 r^{*}, v^{*}$ \\
\hline Pedro Celdrán & & Alcantarilla & a Gines de Cartagena mereader & Murcin & $275 \mathrm{mrs}$. & I vara & pafio negro & S. Juan & $362-F .566 r^{\circ}, v^{\circ}$ \\
\hline Antơn Izqquierdo & & Murcia & Ginés de Cartagena mercader & Murcia & 450 mrs. & $\begin{array}{l}1 \text { vara } \\
1 \text { gabán }\end{array}$ & buriel & S. Juan & $362-\mathrm{F} .567 \mathrm{r}^{\circ}, \mathrm{v}^{*}$ \\
\hline Miguel Sánchez & trnjinero & Murcia & Gincs de Cartagena mercader & Murcia & 450 mrs. & $\begin{array}{l}1 \text { capote } \\
1 \text { vara y media }\end{array}$ & $\begin{array}{l}\text { sayal } \\
\text { buricl f. }\end{array}$ & S. Juan & $362-F .569 r^{\circ}, v^{\circ}$ \\
\hline Alfonso de Toledo & cantarero & Murcia & Salvador Navarro mercader & Murcia & $3.000 \mathrm{mrs}$ & 4 varas & pafto de grana & I atio & 362-F. $570 r^{\circ}, v^{\circ}$ \\
\hline Pedro de Morata & & Murcia & Ginés Cartagena & Murcia & 540 mrs. & $\begin{array}{l}1 \text { vara y cuarto } \\
2 \text { varas y media }\end{array}$ & $\begin{array}{l}\text { palmilla } \\
\text { buricl }\end{array}$ & S. Juan & 362-F. 570r, $v^{\circ}$ \\
\hline Juan Gareia & & Murcia & Gincs de Cartagena mercader & Murcia & $572 \mathrm{mrs}$. & 2 varas y $2 / 3$ & $\begin{array}{l}\text { buriel diecio. } \\
\text { Cuenca }\end{array}$ & S. Juan & 362-F. 573r', $v^{\circ}$ \\
\hline Francisco Celdrán & albardero & Murcia & Bemardino Zamora mercader & Murcia & $690 \mathrm{mrs}$. & 30 varas & jerga & $\begin{array}{l}\text { Carnesto- } \\
\text { lendas }\end{array}$ & 608-F. 180r, $v^{\circ}$ \\
\hline Juan Tomśs & labrador & Aleantarilla & Ginés de Cartagena mercader & Murcja & $238 \mathrm{mrs}$ & 2 varas & $\begin{array}{l}\text { buriel diecio. } \\
\text { forastro. }\end{array}$ & S.Juan & 362-F. 574r", $v^{\circ}$ \\
\hline Juan Calvillo & & Murcia & Gines de Cartagena mereader & Murcia & $600 \mathrm{mrs}$. & 4 varas y media & buriel foraster. & S. Juan & $362-F^{\circ} .577 r^{\circ}, v^{\circ}$ \\
\hline Pedro García & cantarero & Alcantarilla & Gines Roldan & Murcia & $650 \mathrm{mrs}$. & 5 varas & buriel & S. Juan & 608-F. 181r, $v^{*}$ \\
\hline Alonso Martinez & tsajinero & Mlurcia & Ginés de Cartagena mercader & $\begin{array}{l}\text { Murcia } \\
\text { Murcia }\end{array}$ & $\begin{array}{l}770 \text { mrs. } \\
748 \text { mrs. }\end{array}$ & $\begin{array}{l}5 \text { varas } \\
5 \text { varas y media }\end{array}$ & $\begin{array}{l}\text { panto brajulad. } \\
\text { diecioch. } \\
\text { buriel fomstero }\end{array}$ & $\begin{array}{l}\text { S. Juan } \\
\text { S. Juan }\end{array}$ & $\begin{array}{l}\text { 362-F. 580 } r^{\circ}, v^{*} \\
\text { 608-F. } 183 r^{\circ}, v^{*}\end{array}$ \\
\hline Juan Vicent & & Nora & Salvador Navarro & Murcia & 700 mrs. & $\begin{array}{l}1 / 2 \text { vara } \\
4 \text { varas y media }\end{array}$ & $\begin{array}{l}\text { negro } \\
\text { buricl }\end{array}$ & S. Juan & 608-F. 185 $r^{\circ}, v^{\circ}$ \\
\hline Diego López & & La Puebla & Gines de Cartagena mereader & Murcia & 853 mrs. & $\begin{array}{l}1 \text { vara } \\
2 \text { varas y media }\end{array}$ & $\begin{array}{l}\text { morado } \\
\text { buriel }\end{array}$ & Sta. M. & 362-F. 583r, v \\
\hline Alonso Martinez & labrador & Murcia & Gints de Cartagena mercader & Murcia & $584 \mathrm{mrs}$. & 6 varas & cnrojado & & 362-F. 508 $r^{\circ}, v^{\circ}$ \\
\hline
\end{tabular}


VENTAS DE DISTINTOS GENEROS DE PAÑOS - TABLA N.․ V (1506)

\begin{tabular}{|c|c|c|c|c|c|c|c|c|c|c|}
\hline \multirow[b]{2}{*}{ Titular } & \multicolumn{2}{|l|}{ PRADOR } & \multicolumn{3}{|c|}{ VENDEDOR } & \multirow[b]{2}{*}{ Precio } & \multirow[b]{2}{*}{ Cantidad } & \multirow[b]{2}{*}{ Esperie } & \multirow[b]{2}{*}{ Placos } & \multirow[b]{2}{*}{ PROTOCOLO } \\
\hline & Oficio & Vecino & Titslar & Oficio & Vecino & & & & & \\
\hline Alfonso de Andosi. a & armero & Murcia & Ginés de Cartagen & a mercader & Murcia & $1.943 \mathrm{mrs}$. & 9 varas y media & $\begin{array}{l}\text { negro sezeno } \\
\text { forastero }\end{array}$ & S. Juan & 608-F. $188 r^{\circ}, v^{\circ}$ \\
\hline Pedro Martínez & alpargatero & Murcia & Francisco Jufre & genovés & Murcia & $6.375 \mathrm{mrs}$ & 3 varas & veinteno paño & S. Juan & 608-F. $188 v^{\circ}-189$ \\
\hline Pedro Martínez & alpargatero & Murcia & Francisco Jufre & & Murcia & $6.375 \mathrm{mrs}$ & una colcha (5Ds.) & & S. Juan & $608-F .188 v^{\circ}-189$ \\
\hline Pedro Martinez & alpargatero & Murcia & Francisco Jufre & genoves & Murcia & $6.375 \mathrm{mrs}$ & $\begin{array}{l}\text { un cortinaje } \\
\text { (9Ds.) }\end{array}$ & & S. Juan & $608-F .188 v^{\circ}-198$ \\
\hline Francisco Valiente & espadador & Murcia & Gines de Cartagen & a mercader & Murcia & $1.200 \mathrm{mrs}$ & $\begin{array}{l}3 \text { varas } \\
3 \text { varas }\end{array}$ & $\begin{array}{l}\text { negro f. } \\
\text { enrojado }\end{array}$ & S. Juan & 362-F. $585 r^{\circ}, v^{\circ}$ \\
\hline Ginés Fuster & espadador & Murcia & Gines de Cartagen & a mencader & Murcia & $1.200 \mathrm{mrs}$ & $\begin{array}{l}5 \text { varas } \\
2 \text { varas y media }\end{array}$ & $\begin{array}{l}\text { buriel f. } \\
\text { rosa diec. }\end{array}$ & S. Juan & $362-F .586 r^{\circ}, v^{\circ}$ \\
\hline Diego Garcra M. & & Murcia & Beatriz Pérez & & Talavera & $1.000 \mathrm{mrs}$ & 3 varas y $3 / 4$ & panto morado & S. Juan & 608 -F. $191 v^{\circ}-192$ \\
\hline Juan Ballester & & Murcia & Bartolome Coque & notario & Murcia & $500 \mathrm{mrs}$ & 2 varas y media & negro diecioch. & Pascua & $608-196 v^{\circ}-197$ \\
\hline Juan Prieto & & Murcia & Bartolome Coque & notario & Murcia & $1.000 \mathrm{mrs}$. & 5 varas & negro diecioch. & Pascua & $608-F .197 v^{\circ}-198$ \\
\hline Diego Tomás & & Murcia & Ginés Roldán & & Murcia & 1.275 mrs. & 6 varas y cuarta & pafto negro & S. Juan & $608-F .198 v^{\circ}-199$ \\
\hline Pedro Perez & & Murcja & Ginés Rodán & & Murcia & $600 \mathrm{mrs}$. & 5 varas & pardillo & S. Juan & 608-F. $199 r^{\circ}, v^{\circ}$ \\
\hline Alonso López de & & Murcia & Ginés Roldán & & Murcia & $1.040 \mathrm{mrs}$ & $\begin{array}{l}2 \text { varas y cuarta } \\
2 \text { varas } \\
2 \text { varas y cuarta }\end{array}$ & $\begin{array}{l}\text { negro die. } \\
\text { palmilla } \\
\text { pard. sez. }\end{array}$ & S. Juan & $608-F .202 r^{\circ}, v^{\circ}$ \\
\hline Cisneros & & Murcia & Diego Lopez & trapero & Murcia & 833 mrs. & 7 varas & buriel & S. Juan & 608-F. 204v'-205 \\
\hline Francisco Contreras & & Murcia & Juan Gómez & & Murcia & $1.500 \mathrm{mrs}$ & 37 varas y media & lienzo bretaña & S. Juan & $608-F .204 r^{\circ}, v^{\circ}$ \\
\hline Antón Pantrinar & & Murcia & Francisco Gómez & tintorero & Murcia & $6.500 \mathrm{mrs}$ & $\begin{array}{l}2 \text { piczas } \\
2 \text { piezas }\end{array}$ & $\begin{array}{l}\text { veintenos } \\
\text { diecioch. }\end{array}$ & Septiembre & $608-F .205 v^{\circ}-206$ \\
\hline Juan Garre & & Murcia & Franciseo Gómez & tintorero & Murcia & $2.200 \mathrm{mrs}$ & 2 piezas & dieciocheno & Septiembre & 608-F. 206r-207 \\
\hline Miguel Pérez & & Murcia & Ginés Roldén & & Murcia & $250 \mathrm{mrs}$ & 2 varas & buriel & S. Juan & 608-F. $211 r^{\circ}, v^{\circ}$ \\
\hline
\end{tabular}




\section{VENTAS DE DISTINTOS GENEROS DE PAÑOS - TABLA N.' VI (1506-1507)}

\begin{tabular}{|c|c|c|c|c|c|c|c|c|c|}
\hline \multicolumn{2}{|c|}{ COMPRADOR } & \multicolumn{3}{|c|}{ VENDEDOR } & \multirow[b]{2}{*}{ Precio } & \multirow[b]{2}{*}{ Cantidad } & \multirow[b]{2}{*}{ Especie } & \multirow[b]{2}{*}{ Ploces } & \multirow[b]{2}{*}{ PROTOCOLO } \\
\hline Tizular & Verino & Titslar & Offio & Veciso & & & & & \\
\hline Juan Bisbe & Murcia & Gines Rolddn & & Murcia & $325 \mathrm{mrs}$ & 2 varas y media & buriel & Pascua & $608-F .211 v^{*}-212^{\circ}$ \\
\hline Diego de Zamora & Murcia & Domenego Maza & genovés & Murcia & $4.500 \mathrm{mrs}$ & $\begin{array}{l}27 \text { varas } \\
2 \text { piezas }\end{array}$ & $\begin{array}{l}\text { Bretaña } \\
\text { fustán }\end{array}$ & & $608-F .212 v^{*}-213$ \\
\hline Martínez Jovera & Murcia & Francisco Gómez & & Murcia & $1300 \mathrm{mrs}$ & 5 varas & negro veinteno & S. Juan & $608-F .213 r^{\circ}, v^{\circ}$ \\
\hline Juan lbdinez & Murcia & Francisco Gómez & & Murcia & $10.640 \mathrm{mrs}$ & 38 varas & negro veinteno & Navidad & $608 . F .213-214 r^{\circ}$ \\
\hline Juan de Valladolid & Murcia & Juancho Onate & genoves & Murcia & $2.625 \mathrm{mrs}$. & $\begin{array}{l}3 \text { varas y media } \\
24 \text { varas }\end{array}$ & $\begin{array}{l}\text { contmai } \\
\text { Valencia }\end{array}$ & $\begin{array}{l}\text { Sta. M. } \\
\text { agosto }\end{array}$ & $608-F .215 v^{*}-216$ \\
\hline Antón Abcllán & Murcia & Juancho Onate & genoves & Murcia & $1.900 \mathrm{mrs}$ & I vara & $\begin{array}{l}\text { contrai } \\
\text { Valencia }\end{array}$ & S. Juan & $608-F \cdot 215 v^{*}-216$ \\
\hline Juan de Soto & Nora & Juan Loppez de Jara & & Murcia & 792 mrs. & 2 varas y $1 / 3$ & rosado veinteno & oS. Juan & 608-J" $217 r^{\circ}, v^{\circ}$ \\
\hline Fernando Gil. & Murcia & Juan de Medina & boticario & Murcia & $1.125 \mathrm{mrs}$. & 6 varas & $\begin{array}{l}\text { chamelote } \\
\text { negro }\end{array}$ & S. Juan & $608-F .221 v^{\circ}-222$ \\
\hline Alonso de D. Pedro & Murcia & Gonzalo López & & Murcia & $650 \mathrm{mrs}$. & 3 varas $t / 4$ & negro diecioch. & S. Juan & $608-\mathrm{F} .222 \mathrm{r}^{\circ}, \mathrm{v}^{\circ}$ \\
\hline Alonso de Zamora zapatero & Murcia & Fernando Perez & escribano & Murcia & $2000 \mathrm{mrs}$. & 7 varas y medio & veinteno buriel & Sta. M: & 608-F. 219r, $v^{\circ}$ \\
\hline Pedro Raya & Nom & Juan lopez de la J. & & Murcia & $1.038 \mathrm{mrs}$ & $\begin{array}{l}5 \text { varas } \\
1 / 2 \text { vara }\end{array}$ & $\begin{array}{l}\text { buricl } \\
\text { enrojado }\end{array}$ & Pascua & 608-F. 227r -228 \\
\hline Findo de Encinas & Alcantarilt & a Juan Lospez de la J. & & Murciz & $680 \mathrm{mrs}$. & 5 vares & buricl & S. Ju2n & 608-F. 228 $r^{\circ}, v^{*}$ \\
\hline Pedro Moñino & Murcia & Juan Lopez de la J. & & Murcia & $700 \mathrm{mrs}$ & 5 varas & & Pascua & 608-F. 230v" \\
\hline Alon. de Villanueva & Murcia & Gines de Cartagena & mercader & Murcia & $1.500 \mathrm{mrs}$. & 3 varas y media & verde diecioch. & S. Juan & 608-F. 231v", 232 \\
\hline Diego de Escobar & Murcia & Juan Garela de Plas. & & Murcia & $700 \mathrm{mrs}$ & 3 varas y media & diecioch. & S. Juan & 608-F. 232r, $v^{*}$ \\
\hline Juan Amat & Alquerias & Ginés Roldán & & Murcia & $1.460 \mathrm{mrs}$ & 11 varas y modis & buriel & S. Juan & 608-F. 235 $\mathrm{r}^{\circ}, v^{\circ}$ \\
\hline
\end{tabular}


VENTAS DE DISTINTOS GENEROS DE PAÑOS - TABLA N.. VII (1507-1510)

\begin{tabular}{|c|c|c|c|c|c|c|c|c|c|c|}
\hline \multicolumn{3}{|c|}{ COMPRADOR } & \multicolumn{3}{|c|}{ VENDEDOR } & \multirow[b]{2}{*}{ Prrio } & \multirow[b]{2}{*}{ Cantidad } & \multirow[b]{2}{*}{ Especie } & \multirow[b]{2}{*}{ Plaso } & \multirow[b]{2}{*}{ PROTOCOLO } \\
\hline Titular & Oficio & Vecisso & Titular & Oficio & Vecino & & & & & \\
\hline Francisco Tordes. & ladrillero & Murcia & Cristobal Zamora & & Murcia & $3.500 \mathrm{mrs}$ & 90 varas & $\begin{array}{l}\text { Bretaña } \\
\text { agosts }\end{array}$ & Sta. M. & $362-43 r^{\circ}, v^{*}$ \\
\hline Juan Capel & & Murcia & Ginés de Cartagena & mercader & Murcia & $340 \mathrm{mrs}$. & 2 varas & buriel & S. Juan & $362.43 r^{\circ}, v^{*}$ \\
\hline Francisco Galera & & Murcia & Gines de Cartagena & mercader & Murcia & $520 \mathrm{mrs}$ & 4 varas & $\begin{array}{l}\text { buriel } \\
\text { diecioch. }\end{array}$ & S. Juan & $362-50 r^{\circ}, v^{\circ}$ \\
\hline- & & Caravaca & Jacomo Cofia & genoves & Murcia & 7875 mrs. & 16 varas & veinteno negro & 15 meses & $362-53 v^{\circ}-55 r^{*}$ \\
\hline Miguel Navarro & & Murcia & Gints de Cartagena & mercader & Murcia & $550 \mathrm{mrs}$ & $\begin{array}{l}2 \text { vara y media } \\
1 \text { quintal }\end{array}$ & $\begin{array}{l}\text { pardillo } \\
\text { higos }\end{array}$ & & $362.61 r^{\circ}, v^{\circ}$ \\
\hline Fernando Benito & & Murcia & Bartolomé Albacete & eperaile & Murcia & $\begin{array}{l}3 \text { fanegas } \\
\text { trigo }\end{array}$ & 1 vara & veinteno negro & S. Juan & $362-66 r^{\circ}, v^{*}$ \\
\hline Alonso de Galve & & Murcia & Juan García Plasen. & & Murcia & $1200 \mathrm{mrs}$ & 7 varas & enrojado & S. Juan & $608-223 v^{\circ}-224 r^{\circ}$ \\
\hline Pedro González & mercader & Murcia & Domenego Maza & mercader & Murcia & 6.610 mrs. & $\begin{array}{l}6 \text { piezas } \\
1 \text { balón }\end{array}$ & $\begin{array}{l}\text { fustán } \\
\text { papel }\end{array}$ & julio & $608-224 r^{\circ}, v^{\circ}$ \\
\hline Francisco Celdrán & albardero & Murcia & Pedro Monreal & & Murcia & $565 \mathrm{mrs}$ & & & un mes y med & $0608-225 r^{\circ}, v^{\circ}$ \\
\hline Rodrigo Gomariz & & Murcia & Juan Lopez Jara & & Murcia & $650 \mathrm{mrs}$ & & & & $608.225 v^{\circ}-226 \mathrm{r}^{\circ}$ \\
\hline Diego de Morata & & Murcia & Juan López Jara & & Murcia & $1.070 \mathrm{mrs}$ & 3 varas $y$ medi2 & $\begin{array}{l}\text { veinteno rosado } \\
1 / 26 \text { meses }\end{array}$ & 1/2 Pascua & $608-229 r^{\circ}, v^{\circ}$ \\
\hline Leonor de Sevilla & & Murcia & Salvador Navarro & mercader & Murcia & $680 \mathrm{mrs}$ & $\begin{array}{l}3 \text { varas } \\
1 / 2 \text { vara }\end{array}$ & $\begin{array}{l}\text { rosado } \\
\text { diecioch. }\end{array}$ & S. Juan & $608-170 v^{*}-171 r^{\circ}$ \\
\hline Andrés & labrador & Murcia & Francisco Ruiz & trapero & Murcia & $850 \mathrm{mrs}$. & 4 varas & diecioch. & Pascua & $362-3 r^{*}, v^{*}$ \\
\hline Pedro Escalona & & Murcia & Ginés de Cartagena & & Murcia & $2.950 \mathrm{mrs}$ & $\begin{array}{l}1 \text { asno pardo } \\
1 / 2 \text { vara ciudad } \\
6 \text { varas } \\
1 \text { capote de sayal } \\
1 / 2 \text { cahiz cebada }\end{array}$ & $\begin{array}{l}\text { forastero } \\
\text { blanco }\end{array}$ & S. Juan & $362-9 r^{\circ}, v^{\circ}$ \\
\hline Alonso Escribano & & & Bartolome Coque & notario & Murcia & $1.432 \mathrm{mrs}$. & $\begin{array}{l}2 \text { varas y cuarto } \\
3 \text { varas y cuarto } \\
\text { I vara y cuarto }\end{array}$ & $\begin{array}{l}\text { veinteno } \\
\text { buriel } \\
\text { verde }\end{array}$ & $\begin{array}{l}\text { 2/3 Pascua } \\
\text { S. Juan }\end{array}$ & $608-242 v^{\circ}, 243 r^{*}$ \\
\hline
\end{tabular}


VENTAS DE DISTINTOS GENEROS DE PAÑOS - TABLA N. VIII (1510)

\begin{tabular}{|c|c|c|c|c|c|c|c|c|c|c|}
\hline \multicolumn{3}{|c|}{ COMPRADOR } & \multicolumn{3}{|c|}{ VENDEDOR } & \multirow[b]{2}{*}{ Precio } & \multirow[b]{2}{*}{ Cantidad } & \multirow[b]{2}{*}{ Especie } & \multirow[b]{2}{*}{ Plaso } & \multirow[b]{2}{*}{ PROTOCOLO } \\
\hline Titular & Oficio & Vecino & Titular & Oficio & Vecino & & & & & \\
\hline Juan García & hortelano & Murcia & Francisco Ruiz & trapero & Murcia & $1.170 \mathrm{mrs}$. & 5 varas y $1 / 3$ & forastero & agosto & $362-13 r^{\circ}, v^{\circ}$ \\
\hline Alonso Salido & & Murcia & Gines de Cartagena & mercader & Murcia & $1234 \mathrm{mrs}$. & 6 varas y media & buriel & & \\
\hline Bertomena Bleda & viuda & Murcia & Salvador Navarro & mercader & Murcia & 1325 mrs. & $\begin{array}{l}2 / 3 \text { vara } \\
1 / 2 \text { vara } \\
3 / 4 \text { vara }\end{array}$ & $\begin{array}{l}\text { fustán } \\
\text { venteno } \\
\text { venteno }\end{array}$ & S. Juan & $603-8 r^{\circ}, v^{*}$ \\
\hline Chinchillano & & Murcia & Salvador Navarro & mercader & Murcia & $\begin{array}{l}5 \text { ducados } \\
\mathrm{Au}\end{array}$ & 2 varas y media & $\begin{array}{l}\text { contrai } \\
\text { Valencia }\end{array}$ & 8 meses & $603-12 r^{\circ}, v^{\circ}$ \\
\hline Francisco Moratón & & Murcia & Salvador Navarro & mercader & Murcia & $15.750 \mathrm{mrs}$. & 21 varas & contrai & 8 meses & $603-26 r^{\circ}, v^{\circ}$ \\
\hline Alonso Vergonoz & & Murcia & Salvador Navarro & mercader & Murcia & $\begin{array}{l}7 \text { ducados } \\
\text { Au }\end{array}$ & 3 varas y media & contrai & S. Miguel & $603-31 r^{\circ}, v^{*}$ \\
\hline Fernando Sánchez & & Murcia & Salvador Navarro & mercader & Murcia & $2.875 \mathrm{mrs}$ & $\begin{array}{l}3 \text { varas y media } \\
3 \text { varas }\end{array}$ & $\begin{array}{l}\text { veinteno } \\
\text { ruande } \mathrm{V} \text {. }\end{array}$ & $\begin{array}{l}\text { Sta. M." } \\
\text { agosto }\end{array}$ & $603-39 r^{*}, v^{*}$ \\
\hline Alonso de Pedriffá & injurado & Murcia & Salvador Navarro & mereader & Murcia & $5.225 \mathrm{mrs}$ & 9 varas y media & veintedoseno & S. Juan & $603-44 r^{\circ}, v^{*}$ \\
\hline Francisco Abellán & & Jumilla & Salvador Navarro & mercader & Murcia & $1.080 \mathrm{mrs}$ & 4 varas y media & veinteno negro & S. Juan & $603-46 r^{\circ}, v^{\circ}$ \\
\hline Gints de Fulleda & & Murcia & Salvador Navarro & mercader & Murcia & $3350 \mathrm{mrs}$. & $\begin{array}{l}1 \text { vara y cuarta } \\
3 \text { varas y cuarto } \\
1 / 2 \text { vara raso }\end{array}$ & $\begin{array}{l}\text { grana } \\
\text { veinteno V. }\end{array}$ & S. Juan & $603-47 r^{\circ}, v^{*}$ \\
\hline Juan de Morcillo & & Molina & Salvador Navarro & mercader & Murcia & $2.700 \mathrm{mrs}$ & $\begin{array}{l}2 \text { varas y cuarta } \\
3 \text { varas y media }\end{array}$ & $\begin{array}{l}\text { ruan } V . \\
\text { veinteno }\end{array}$ & $\begin{array}{l}\text { Todos los } \\
\text { Santos }\end{array}$ & $603-48 r^{\circ}, v^{\circ}$ \\
\hline Juan de Sta. Cruz & & Molina & Salvador Navarro & mercader & Murcia & $375 \mathrm{mrs}$. & $3 / 4$ varas & ruan de V. & S. Miguel & $603.49 r^{\circ}, v^{*}$ \\
\hline Rodrigo de Aledo & & Aledo & Salvador Navarro & mercader & Murcia & 1.904 mrs. & 8 vams & diecioch. verde & S. Miguel & $603-51 r^{*}, v^{*}$ \\
\hline Pedro de Manresa & & Murcia & Salvador Navarro & mercader & Murcia & $1.540 \mathrm{mrs}$. & 7 varas & diecioch. & Navidad & $603-55 r^{\circ}, v^{\circ}$ \\
\hline Juan de Torres & & Murcia & Salvador Navarro & mercader & Murcia & $990 \mathrm{mrs}$. & 4 varas y media & diecioch. & Navidad & $603-56 \mathrm{r}^{\circ}, \mathrm{v}^{*}$ \\
\hline Gil de Burgos & & Cotillas & Salvador Navarro & mercader & Murcia & $3337,5 \mathrm{mrs}$ & $\begin{array}{l}.4 \text { varas y media } \\
5 \text { varas }\end{array}$ & $\begin{array}{l}\text { veinteno } \\
\text { veintedos. }\end{array}$ & $\begin{array}{l}\text { Todos los } \\
\text { Santos }\end{array}$ & $605-57 r^{\circ}, v^{\circ}$ \\
\hline
\end{tabular}


VENTAS DE DISTINTOS GENEROS DE PAÑOS - TABLA N.․ IX (1510)

\begin{tabular}{|c|c|c|c|c|c|c|c|c|c|}
\hline \multicolumn{2}{|l|}{ COMPRADOR } & \multicolumn{3}{|c|}{ VENDEDOR } & \multirow[b]{2}{*}{ Precio } & \multirow[b]{2}{*}{ Cantidad } & \multirow[b]{2}{*}{ Especie } & \multirow[b]{2}{*}{ Plaseo } & \multirow[b]{2}{*}{ PROTOCOLO } \\
\hline Titular & Vecino & Titular & Oficio & Vecino & & & & & \\
\hline Francisco Martínez carpintero & Murcia & Salvador Navarro & mereader & Murcia & $1.547 \mathrm{mrs}$ & 7 varas & diecioch. & $\begin{array}{l}\text { 1/2 Navidad } \\
1 / 2 \text { Carnestol. }\end{array}$ & $603-61 r^{\circ}, v^{\circ}$ \\
\hline Alonso de Pedriñán jurado & Murcia & Salvador Navarro & mercader & Murcia & $4862,5 \mathrm{mrs}$ & $\begin{array}{l}3 \text { varas y } 3 / 4 \\
3 \text { varas y media }\end{array}$ & $\begin{array}{l}\text { ruan } \\
\text { contrai }\end{array}$ & Navidad & $603-63 r^{*}, v^{\circ}$ \\
\hline Juan Perellón & Murcia & Salvador Navarro & mercader & Murcia & $630 \mathrm{mrs}$. & 4 varas $y$ media & $\begin{array}{l}\text { buriel } \\
\text { diecioch. }\end{array}$ & $\begin{array}{l}\text { Todos los } \\
\text { Santos }\end{array}$ & $603-64 r^{\circ}, v^{\circ}$ \\
\hline Diego de Burgos & Cotillas & Salvador Navarro & mercader & Murcia & 700 mrs. & 5 varas & buriel & Navidad & $603-66 \mathrm{r}^{\circ}, \mathrm{v}^{\circ}$ \\
\hline Francisco Guerrero labrador & Murcia & Francisco Ruiz & trapero & Murcia & $1.600 \mathrm{mrs}$ & $\begin{array}{l}20 \text { varas } \\
3 \text { varas y media }\end{array}$ & $\begin{array}{l}\text { colores } \\
\text { Londres }\end{array}$ & Navidad & $362-487 r^{\circ}-v^{\circ}$ \\
\hline$\longrightarrow$ & Alcantarilla & Salvador Navarro & mercader & Murcia & 777 mrs. & 2 varas y cuarto & veinteno negro & Pascua & $603-70 r^{\circ}, v^{\circ}$ \\
\hline Alon. de Andosilla & Murcia & Salvador Navarro & mercader & Murcia & $2.555 \mathrm{mrs}$ & 7 varas & veinteno & Camestol. & $603.71 r^{\circ}, v^{*}$ \\
\hline Pedro Ruiz & Murcia & Salvador Navarro & mercader & Murcia & $1.500 \mathrm{mrs}$ & & & Pascua & $603-76 r^{\circ}, v^{\circ}$ \\
\hline Alon. de Vergonoz & Murcia & Salvador Navarro & mercader & Murcia & $4.500 \mathrm{mrs}$ & 6 varas & contrai & S. Juan & $603-77 r^{\circ}, v^{\circ}$ \\
\hline Martin de Alhama & Aledo & Salvador Navarro & mercader & Murcia & $1.250 \mathrm{mrs}$ & 2 varas y media & veinteno & & $603-78 r^{\circ}, v^{\circ}$ \\
\hline Alonso Román & Jumilla & Salvador Navarro & mercader & Murcia & $3.750 \mathrm{mrs}$ & 3 varas y cuarto & contraj & Pascua & $603-75 r^{\circ}, v^{*}$ \\
\hline Diego de Molares & Murcia & Salvador Navarro & mercader & Murcia & $2.650 \mathrm{mrs}$ & 56 varas & naval & 8 meses & $603-79 \mathrm{r}^{\circ}, \mathrm{v}^{*}$ \\
\hline Jimènez & Murcia & Salvador Navarro & mercader & Murcia & $3.150 \mathrm{mrs}$ & 7 varas & raso negro & Pascua & $603-80 r^{\circ}, v^{\circ}$ \\
\hline Catalina Puxverte viuda & Murcia & Francisco Ruiz & trapero & Murcia & $750 \mathrm{mrs}$. & 1 vara y media & veinteno negro 1 & Navidad & $362-512 r^{\circ}, v^{\circ}$ \\
\hline Diego de Aledo & Lorqui & Salvador Navarro & mercader & Murcia & $1.700 \mathrm{mrs}$ & 3 varas & grana Valencia 1 & Navidad & $603-81 r^{*}, v^{*}$ \\
\hline Joaquin Garcia & Murcia & Francisco Ruiz & trapero & Murcia & $874 \mathrm{mrs}$. & 3 varas y $2 / 3$ & $\begin{array}{l}\text { diecioch. } \\
\text { palmilla }\end{array}$ & Navidad & $362-524 r^{\circ}, v^{\circ}$ \\
\hline Pedro Aledo & Aledo & Salvador Navarro & mercader & Murcia & 10.530 mrs. & $\begin{array}{l}12 \text { varas } \\
7 \text { varas }\end{array}$ & $\begin{array}{l}\text { veintedos. } \\
\text { contrai }\end{array}$ & & $603-83 r^{\circ}, v^{\circ}$ \\
\hline
\end{tabular}


VENTAS DE DISTINTOS GENEROS DE PAÑOS - TABLA N.․ X (1510)

\begin{tabular}{|c|c|c|c|c|c|c|c|c|c|c|}
\hline \multicolumn{3}{|c|}{ COMPRADOR } & \multicolumn{3}{|c|}{ VENDEDOR } & \multirow[b]{2}{*}{ Precio } & \multirow[b]{2}{*}{ Cantidad } & \multirow[b]{2}{*}{ Especie } & \multirow[b]{2}{*}{ Plazo } & \multirow[b]{2}{*}{ PROTOCOLO } \\
\hline Titular & Oficio & Vecino & Tirular & Oficio & Vecino & & & & & \\
\hline Juan Tenza & & Murcia & Salvador Navarro & mercader & Murcin & 7.500 mrs. & 10 varas & contrai & 8 meses & $603-83 r^{\circ}, v^{\circ}$ \\
\hline Juan Murcia & & Murcia & Pedro Castellon & peraile & Murcia & 350 mrs. & 2 baras y media & buriel diecioch. & Navidad & $362-531,532$ \\
\hline Cristóbal Dasta & & Murcia & Salvador Navarro & mereader & Murcia & $2200 \mathrm{mrs}$ & 4 varas & ruan plateado & 8 meses & $603-84 r^{\circ}, v^{\circ}$ \\
\hline Pascual García & & $\begin{array}{l}\text { L.ugar } \\
\text { A. Fajardo }\end{array}$ & Salvador Navarro & mercader & Murcia & $3060 \mathrm{mrs}$ & 10 varas & $\begin{array}{l}\text { veinteno } \\
\text { verde limonado }\end{array}$ & Pascua & $603-85 r^{*}, v^{\circ}$ \\
\hline Diego de Morata & & Murcia & Salvador Navarro & mercader & Murcia & 1.400 mrs. & 4 varas & veinteno negro & Pascua & $603.86 r^{\circ}, v^{*}$ \\
\hline Juan Fuentes & & Murcia & Salvador Navarro & mercader & Murcia & 24 rs. plata & 6 varas & buricl & S. Juan & $603-87 r^{\circ}, v^{\circ}$ \\
\hline Juan Enriquez & labrador & Murcia & Pedro Castillo & peraile & Murcia & $1.190 \mathrm{mrs}$. & 9 varas y cuarto & diecioch. buriel & Navidad & $362-562,563$ \\
\hline Bartolome Dlaz & & Murcia & Salvador Navarro & mercader & Murcia & 990 mrs. & 4 varas y media & diecioch burial & S. Juzn & $603-89 r^{\circ}, v^{*}$ \\
\hline Juan Garcla & & Murcia & Salvador Navarro & mercader & Murcia & $1.840 \mathrm{mrs}$ & 8 varas & diecioch ciudad & IS. Juan & $603-92 r^{\circ}, v^{\circ}$ \\
\hline Alonso Fontes & & Murcia & Salvador Navarro & mercader & Murcia & $5.175 \mathrm{mrs}$ & 9 varas & $\begin{array}{l}\text { veintedo. } \\
\text { Valencia }\end{array}$ & S. Juan & $603-93 r^{\circ}, v^{*}$ \\
\hline Teresa de Cascales & viuda & Murcia & Salvador Navarro & mercader & Murcia & 2.210 mrs. & 10 varas & diecioch. negro & S. Juan & $603-94 r^{\circ}, v^{\circ}$ \\
\hline Pedro Teruel & & Murcia & Salvador Navarto & mercader & Murcia & $3.337 \mathrm{mrs}$ & 3 varas y media & & Pascua & 603-95r, $v^{*}$ \\
\hline Alonso Tirado & & Murcia & Salvador Navarro & mercader & Murcia & $2600 \mathrm{mrs}$ & $\begin{array}{l}2 / 3 \text { vara } \\
3 \text { varas } y \text { media } \\
1 / 2 \text { vara }\end{array}$ & $\begin{array}{l}\text { morado } \\
\text { rosado } \\
\text { verde }\end{array}$ & Pascua & $603-96 r^{\circ}, v^{\circ}$ \\
\hline Juan Fernández & & Murcia & Salvador Navarro & mercader & Murcia & $1.450 \mathrm{mrs}$ & 6 varas & $\begin{array}{l}\text { veintedos. } \\
\text { Valencia }\end{array}$ & S. Juan & 603-97 $\mathrm{r}^{\circ}, \mathrm{v}^{*}$ \\
\hline Pedro Vidal & & Puebla & Salvador Navarro & mercader & Murcia & 773 mrs. & & diecioch. & S. Juan & $603-98 r^{\circ}, v^{\circ}$ \\
\hline & viuda & Murcia & Salvador Navarro & mercader & Murcia & $5.458 \mathrm{mrs}$. & $\begin{array}{l}1 / 3 \text { vara } \\
3 \text { varas } y 1 / 2\end{array}$ & $\begin{array}{l}\text { contrai } \\
\text { grano }\end{array}$ & S. Juan & 603-99r, $v^{\circ}$ \\
\hline
\end{tabular}




\section{VENTAS DE DISTINTOS GENEROS DE PAÑOS - TABLA N.. XI (1510-1514)}

\section{COMPRADOR}

Titular

$$
\text { Oficio }
$$

Pedro de Avila

Teresa de Alarcón viuda

Juan de Munuera

Francisco Martin

Pedro Tomás

Pedro Carrillo

Francisco Zapata

Francisco Pintado

Diego Tomás

Pedro Blasco

Francisco Pagán

Fernando Martínez herrero

Isabel Pérez

Fernando Garcla

Juan Aranda

Pedro Angosto

Juan Miñano

\section{VENDEDOR}

Vocino Tisular Oficio Vecino

Alcantarilla Salvador Navarro mercader Murcia

Murcia Salvador Navarro mercader Murcia

Murcia Salvador Navarro mercader Murcia

Blanca Salvador Navarro mercader Murcia

Blanca Salvador Navarro mercader Murcia

Puebla Salvador Nayarro mercader Murcia

Puebla Salvador Navarro mercader Murcia

Librilla Salvador Navarro mercader Murcia

Murcia Salvador Navarro mereader Murcia

Fortuna Salvador Navarro mercader Murcia

Molina Salvador Navarro mercader Murcia

Murcia Salvador Navarro mercader Murcia

Murcia Salvador Navarro mercader Murcia

Puebla Salvador Navarro mercader Murcia

Murcia Salvador Navarro mercader Murcia

Ricote Salvador Navarro mercader Murcia

Ricote

\section{Precio Cantidad}

6.000 mrs. 8 varas

600 mrs. 3 varas

$945 \mathrm{mrs}$. $3 / 4$ vara

3 varas y media

$2.145 \mathrm{mrs} .1$ vara

3 varas

$1.098 \mathrm{mrs} .1$ vara

3 varas y media

$1 / 2$ vara

773 mrs. 2 varas

1 vara

$1 / 2$ vara

$703 \mathrm{mrs} . \quad 1 / 2$ vara

3 varas

$774 \mathrm{mrs}$. 3 varas y media verde oscuro

774 mrs. 3 varas $y$ media

$996 \mathrm{mrs} .2$ varas y cuarto

$1 / 4$ vara

655 mrs. 2 varas y $3 / 4$

3.036 mrs. 6 varas y media 4 varas

1336 mrs. 3 varas y $1 / 4$ $3 / 4$ vara

663 mrs. 3 varas

2600 mrs. 3 varas y media

875 mrs. 3 varas y media

$553 \mathrm{mrs} .2$ varas $y$ media
Esperis

contral

verde claro

veinteno

diecioch.

veintedos, S. Juan

rosado

diecioch.

verde

blanqueta

diecioch.

amarilla

negra

blanqueta

verde

diecioch

morado

veinteno

colorado

color

diecioch.

veintesen.

diecioch.

contrai

tenido

contrai

veinteno

morado

Place

Sta. M. agosto

S. Juan

S. Juan

S. Juan

S. Juan

S. Juan

Pascua

S. Juan

S. Juan

pascua

S. Juan

S. Juan
$603-108 r^{\circ}, v^{\circ}$

Santa M.a

603.110r, v

Santa M.s

$603.111 r^{\circ}, v^{\circ}$

$603-112 r^{\circ}, v^{\circ}$

603-113r, v $v^{\circ}$

603-114r, $v^{\circ}$

$603-115 r^{\circ}, v^{\circ}$

$603.116 r^{\circ}, v^{\circ}$ 


\section{VENTAS DE DISTINTOS GENEROS DE PAÑOS - TABLA N. XII (1514)}

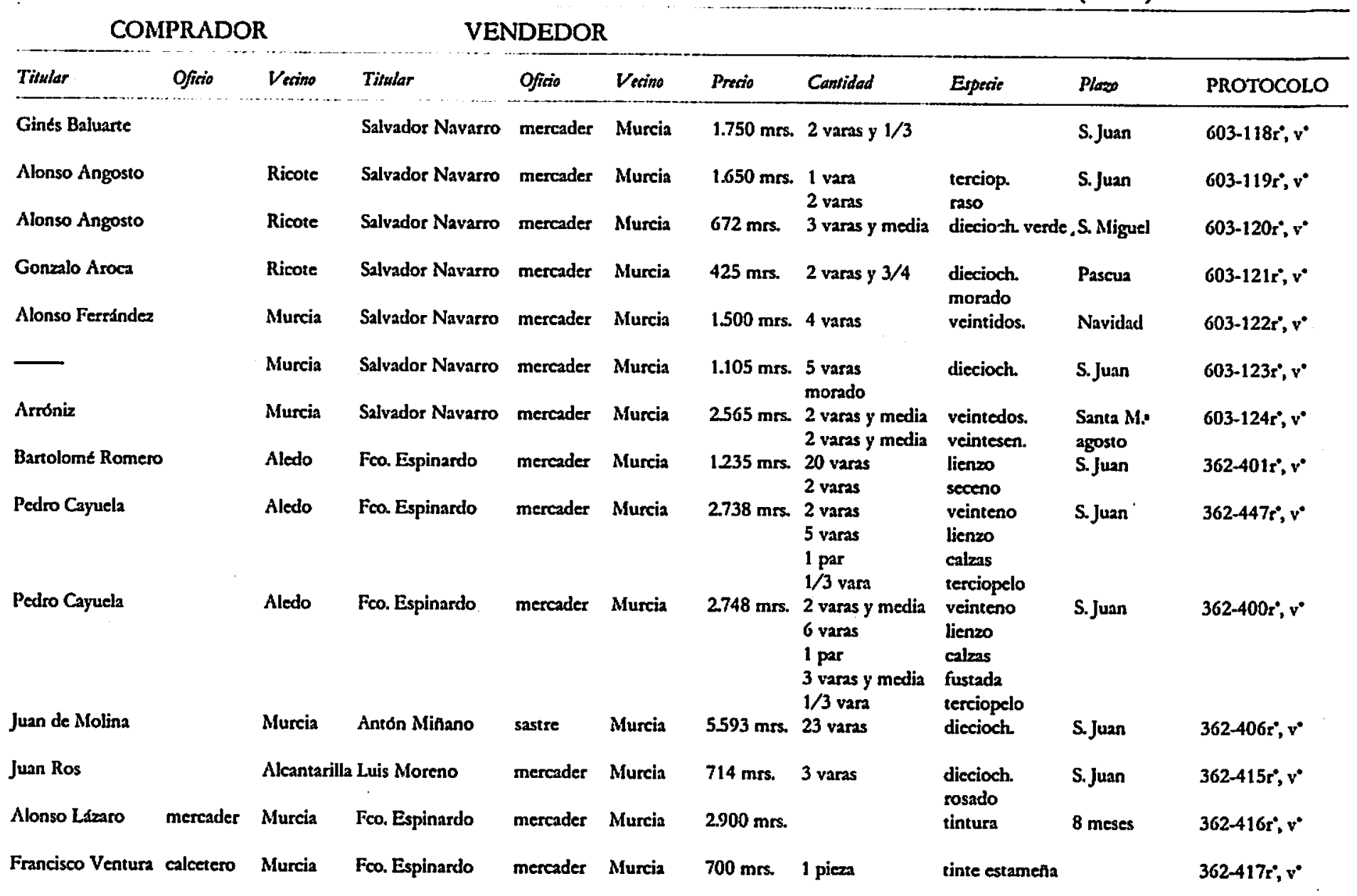




\section{VENTAS DE DISTINTOS GENEROS DE PAÑOS - TABLA N.: XIII (1514)}

\begin{tabular}{|c|c|c|c|c|c|c|c|c|c|c|}
\hline \multicolumn{3}{|c|}{ COMPRADOR } & \multicolumn{3}{|c|}{ VENDEDOR } & \multirow[b]{2}{*}{ Precio } & \multirow[b]{2}{*}{ Cantidad } & \multirow[b]{2}{*}{ Espesie } & \multirow[b]{2}{*}{ Plo0so } & \multirow[b]{2}{*}{ PROTOCOLO } \\
\hline Tirular & Oficio & Vecino & Tisular & Oficio & Vecino & & & & & \\
\hline Juan de Costeta & peraile & Murcia & Fco. Espinardo & mercader & Murcia & $2.200 \mathrm{mrs}$ & & tintes & S. Juan & $362-419 r^{\circ}, v^{\circ}$ \\
\hline Guillén Rives & peraile & Murcia & Fco. Espinardo & mercader & Murcia & $1.100 \mathrm{mrs}$ & & tintes & $\begin{array}{l}\text { Todos los } \\
\text { Santos }\end{array}$ & $362-423 r^{\circ}, v^{*}$ \\
\hline Pedro Gomariz & peraile & Murcia & Fco. Espinardo & mercader & Murcia & $1.500 \mathrm{mrs}$ & & & 8 meses & $362-435 r^{\circ}, v^{*}$ \\
\hline Alonso Perez & zapatero & Murcia & Fco. Espinardo & mercader & Murcia & 1.237 mrs. & $\begin{array}{l}2 \text { varas y } \\
3 \text { palmos }\end{array}$ & veintedo. $\mathrm{r}$ & S. Miguel & $362-689 r^{\circ}, v^{*}$ \\
\hline Pedro Clavero & tundidor & Murcia & Fco. Espinardo & mercader & Murcia & $5.200 \mathrm{mrs}$ & & tintes & 8 meses & $362-449 r^{\circ}, v^{*}$ \\
\hline Ginés de la Riva & labrador & Murcia & Antón Miñao & sastre & Murcia & $4.040 \mathrm{mrs}$ & 17 varas & diecioch. & S. Juan & $362-440 r^{\circ}, v^{*}$ \\
\hline Ginés Fuster & & Murcia & Luis Moreno & mercader & Murcia & 3.344 mrs. & $\begin{array}{l}3 \text { varas } \\
3 \text { varas y media }\end{array}$ & $\begin{array}{l}\text { veinteno } \\
\text { veintedo. }\end{array}$ & Pascua & $362-497 r^{\circ}, v^{\circ}$ \\
\hline Rodrigo Sevillano & procusador & Murcia & Alonso Celdrán & jurado & Murcia & $4.500 \mathrm{mrs}$. & 6 varas & contrai & 9 meses & $362-408 r^{\circ}, v^{\circ}$ \\
\hline Juan Garcia & & Cartagena & Luis Moreno & mercader & Murcia & 5.421 mrs. & $\begin{array}{l}22 \text { varas y } \\
3 \text { palmos }\end{array}$ & diecioch. & Santa M. & $362-696 r^{\circ}, v^{*}$ \\
\hline Martín Tamayo & & Murcia & Luis Moreno & mercader & Murcia & $490 \mathrm{mrs}$ & 3 varas y $1 / 4$ & buriel & S. Juan & $362-680 r^{\circ}, v^{\circ}$ \\
\hline
\end{tabular}


COMERCIO DE LA LANA - TABLA N. XIV (1506-1515)

\begin{tabular}{|c|c|c|c|c|c|c|c|c|c|c|}
\hline \multicolumn{3}{|c|}{ COMPRADOR } & \multicolumn{3}{|c|}{ VENDEDOR } & \multirow[b]{2}{*}{ Precio } & \multirow[b]{2}{*}{ Cansided } & \multirow[b]{2}{*}{ Especie } & \multirow[b]{2}{*}{ Plosesp } & \multirow[b]{2}{*}{ PROTOCOLO } \\
\hline Titular & Ofritio & Vecino & Titular & Oficio & Vecino & & & & & \\
\hline Maestre Alonso & guantero & Murcia & Jaime Espana & & Ciezn & $2.508 \mathrm{mrs}$. & 114 libras & & Pascua & $608-107 r^{\circ}, v^{*}$ \\
\hline Ginés Cartagena & mereader & Murcja & Lotenzo & & Murcia & $12.500 \mathrm{mrs}$ & 30 atrobas & lana blanea & $\begin{array}{l}\text { 1/2 S. Juan } \\
\text { l/2 Sta. M.: }\end{array}$ & $362-532 r^{\circ}, v^{*}$ \\
\hline Maestre Alonso & guantero & Murcia & Juan de Castro & & Murcia & $4.725 \mathrm{mrs}$. & 9 arrobas & & $\begin{array}{l}\text { 1/2 Carnestol. } \\
1 / 2 \text { Pascua }\end{array}$ & $608-202 v^{\circ}, 203 r^{\circ}$ \\
\hline Maestre Alonso & guantero & Murcia & & & Albacete & $3.344 \mathrm{mrs}$. & & & Cameștol & $608-218 r^{\circ}, 219$ \\
\hline Maestre Alonso & guantero & Murcia & Martin Blanco & & Murcia & $1.575 \mathrm{mrs}$. & 3 arrobas & & Pascua & $608-221 r^{\circ}, v^{\circ}$ \\
\hline Felipe Escaja & genoves & Murcia & Andrés Carasco & & Caravaca & $\begin{array}{l}420 \mathrm{mrs} . \\
2 \pi 5 .\end{array}$ & $\begin{array}{l}\text { Toda la lana } \\
\text { de su ganado }\end{array}$ & $\begin{array}{l}\text { blanca } \\
\text { fina }\end{array}$ & $\begin{array}{l}1 / 2 \text { al esquil. } \\
1 / 2 \text { VII-1508 }\end{array}$ & $362-51 r^{\circ}, v^{*}$ \\
\hline Trancisco Maynetz & & Murcia & jaime Mariner & & Caravaca & $\begin{array}{l}300 \text { mrs. } \\
\text { arr. }\end{array}$ & 130 arrobas & blanca & enero & $362-476 v^{\circ}, 478 r^{\circ}$ \\
\hline Juan Vázquez & & Baza & Esteban Lopez & & Murciz & $325 /$ arroba & 150 arrobas & blanca merina & & $363-458 v^{*}, v^{*}$ \\
\hline
\end{tabular}


COMERCIO DE GANADO - TABLA N. XV (1506-1507)

\begin{tabular}{|c|c|c|c|c|c|c|c|c|c|}
\hline \multicolumn{2}{|c|}{ COMPRADOR } & \multicolumn{3}{|c|}{ VENDEDOR } & \multirow[b]{2}{*}{ Precio } & \multirow[b]{2}{*}{ Cantidad } & \multirow[b]{2}{*}{ Especie } & \multirow[b]{2}{*}{ Plazso } & \multirow[b]{2}{*}{ PROTOCOLO } \\
\hline Titwlar & Vecino & Tirular & Oficio & Vecino & & & & & \\
\hline Alonso Almena & Alcantarilla & a Fernando Santa Fe & & Murcia & $\begin{array}{l}\text { un cahiz } \\
\text { de trigo }\end{array}$ & 1 yegua & castanla & S. Juan & \\
\hline Bartol. Escribano & Murcia & Alonso Femández & & Murcia & $2.700 \mathrm{mrs}$ & I potro & castafio & $\begin{array}{l}\text { Santa M.: } \\
\text { agosto }\end{array}$ & $608-91,92$ \\
\hline Francisco Contreras & Murcia & Alonso García & & Murcia & $1800 \mathrm{mrs}$ & 1 rocin & & S. Miguel & $608-127 r^{\circ}, v^{*}$ \\
\hline Juan Alvarez & Murcia & Francisco Soriano & & Murcia & $2000 \mathrm{mrs}$ & 1 mula & prieta & S. Juan & $608-178 v^{\circ}, 179 v^{\circ}$ \\
\hline Pedro Lopez & Abanilla & Fernando Jimenez & & Hellin & $4.975 \mathrm{mrs}$ & 1 macho & cerril & 1 año & $362-575 r^{\circ}, v^{*}$ \\
\hline Rodrigo Aledo & Abanilla & Fernando Jiménez & & Hellin & $4.125 \mathrm{mrs}$ & 1 macho & cerril & 1 afio & $362-576 r^{\circ}, v^{\circ}$ \\
\hline Lorenzo Avilés & Abanilla & Fernando Jimenez & & Hellin & $3.000 \mathrm{mrs}$ & 1 macho & alberda & S. Juan & $362-579 r^{\circ}, v^{\circ}$ \\
\hline Juan Calafate & Cartagena & Fernando Jimtrnez & & Hellin & $4300 \mathrm{mrs}$. & 1 macho & cerril & 1 año & $362-584 r^{\circ}, v^{*}$ \\
\hline Diego Garcfa & Murcia & Fernando Jiménez & & Hellin & $3.900 \mathrm{mrs}$. & 1 mula & pardilla & $\begin{array}{l}\text { Santa M.. } \\
\text { agosto }\end{array}$ & $362-587 r^{\circ}, v^{*}$ \\
\hline Juan de Balibrea & Murcia & Fernando Jiménez & & Hellin & $3.700 \mathrm{mrs}$. & 1 macho & cerril nucio & S. Juan & $364-86 r^{\circ}, v^{\circ}$ \\
\hline Pedro de Enguera & Murcia & Pedro López & aserrador & Murcia & $3.925 \mathrm{mrs}$. & 1 bucy & & S. Juan & $608-194 v^{\circ}, 195 r^{\circ}$ \\
\hline Mateo de Quesada & Murcia & Juan Vargonoz & & Murcia & $834 \mathrm{mrs}$. & 1 burra & & S. Juan & $608-169,170$ \\
\hline Luis Carles & Murcia & Martin Lopez & & Murcia & $3.500 \mathrm{mrs}$ & 1 buey & & Marzo & $608-195 v^{\circ}, 196 v^{\circ}$ \\
\hline Fco. de Contreras & Murcia & Francisco Cardona & & Murcia & 1.620 mrs. & 1 yegua & color morcillo & Navidad & $608-201 r^{*}, v^{*}$ \\
\hline Juan de Torres & Murcia & Juan Rey & genovés & Murcia & $1.125 \mathrm{mrs}$ & 1 asno & & sin concretar & $608-210 r^{\circ}, v^{*}$ \\
\hline Alonso de Córdoba & Murcia & Gines de Cartagena & mercader & Murcia & 1.100 mrs. & 1 rocin & & S. Juan & $608-209 r^{\circ} v^{\circ}$ \\
\hline
\end{tabular}


COMERCIO DE GANADO - TABLA N. XVI (1507)

\begin{tabular}{|c|c|c|c|c|c|c|c|c|c|c|}
\hline \multicolumn{3}{|c|}{ COMPRADOR } & \multicolumn{3}{|c|}{ VENDEDOR } & \multirow[b]{2}{*}{ Precio } & \multirow[b]{2}{*}{ Contided } & \multirow[b]{2}{*}{ Esperie } & \multirow[b]{2}{*}{ Plaso } & \multirow[b]{2}{*}{ PROTOCOLO } \\
\hline Tirular & Oficio & Vectino & Titular & Oficio & Vecino & & & & & \\
\hline Pedro Martinez & & Murcia & Andres Soriano & & Murcia & $1200 \mathrm{mrs}$ & 1 asno & & Santa M. & $608-171 r^{\circ}, 172$ \\
\hline Juan Cruzado & & La Raya & Femando Garcla & & Murcia & $612 \mathrm{mrs}$. & 1 asno & & Santa M. & $608-234 v^{\circ}, 235 r^{\circ}$ \\
\hline Fco. Hernández & & Murcia & Antonio Angulo & $\begin{array}{l}\text { Escribano } \\
\text { Inquisic. }\end{array}$ & Murcia & $3.600 \mathrm{mrs}$ & 1 caballo & castafio & $\begin{array}{l}\text { Camestol. } \\
\text { S. Juan }\end{array}$ & $562-27 r^{\circ}, v^{\circ}$ \\
\hline Francisca Montreal & viuda & Murcia & Juan Bermejo & $\begin{array}{l}\text { cristiano } \\
\text { nuevo }\end{array}$ & Alcantarilla & $2000 \mathrm{mrs}$ & 1 ycgua & Castafia & & $362-32 r^{\circ}, v^{*}$ \\
\hline Pedro Martínez & criado & Murcia & Juan de Murcia & marchante & Murcia & $1.300 \mathrm{mrs}$ & 1 asno rocín & & S. Juan & $608-240 r^{\circ}, v^{\circ}$ \\
\hline Garcia Requena & & Requena & Bartolome Coque & notario & Murcia & $1.900 \mathrm{mrs}$ & 1 asno & & S. Juan & $608-240 r^{\circ}, v^{*}$ \\
\hline Juan Ruiz & & Murcia & Andres Soriano & & Murcia & $1.250 \mathrm{mrs}$. & 1 asno & & Santa M." & $608-241 r^{\circ}, v^{\circ}$ \\
\hline F. de Parcja & & Mula & Francisco Manresa & & Murcia & $2750 \mathrm{mrs}$. & 1 potro cerril & castano & $\begin{array}{l}\text { Todos los } \\
\text { Santos }\end{array}$ & $362-496 r^{\circ}, v^{\circ}$ \\
\hline Alonso Ferrández & & Mula & Mareo Ferránder & & Moratalla & $6.500 \mathrm{mrs}$. & 500 ovejas & & 4 años & $362-517 r^{\circ}, v^{\circ}$ \\
\hline Pedro Martínez & & Murcia & Fdo. de Villena & $\begin{array}{l}\text { racionero } \\
\text { de la Igiesia } \\
\text { bachiller }\end{array}$ & Murcia & 6 Ducados & 1 asno & & Navidad & $362-530 r^{\circ}, v^{\circ}$ \\
\hline Fernando García & labrador & Puebla & Alonso Carrillo & labrador & Puebla & $\begin{array}{l}31 / 2 \mathrm{Du}- \\
\text { cados }\end{array}$ & 1 potro cerril & morcillo & S. Juan & $362-536 v^{\circ}, 537 v^{\circ}$ \\
\hline Antón Puxverte & & Murcia & Alonso Gómez & tintorero & Murcia & $1.570 \mathrm{mrs}$ & 1 potro & alazan & Carnestol. & $362-561 v^{\circ}, 562 r^{\circ}$ \\
\hline Alonso Garcia & & Murcia & Gonzalo Soto & $\begin{array}{l}\text { fiscal } \\
\text { iglesia }\end{array}$ & Murcia & $1.500 \mathrm{mrs}$ & 1 asno & Pascua & Pascua & $362-446 r^{\circ}, v^{\circ}$ \\
\hline Juan Martinez & & Murcia & Luis Madrid & mercader & Valencia & $4.500 \mathrm{mrs}$. & 1 mula & & S. Miguel & $362.707 r^{\circ}, v^{\circ}$ \\
\hline Gómex Peñaranda & & Murcia & Luis Madrid & mercader & Valencia & $7.500 \mathrm{mrs}$ & $1 \mathrm{mula}$ & & S. Juan & $362-709 \mathrm{r}^{\circ}, \mathrm{v}^{*}$ \\
\hline Pedro Horgiles & & & Luis Madrid & mercader & Valencia & $5.500 \mathrm{mrs}$. & 1 mula & & S. Juan & $362-708 r^{\circ}, v^{\circ}$ \\
\hline Pedro Piñar & & & Luis Madrid & mercader & Valencia & $6.500 \mathrm{mrs}$. & $1 \mathrm{mula}$ & & 1 aก็น & $362-713 r^{\circ}, v^{\circ}$ \\
\hline
\end{tabular}




\section{COMERCIO DE GANADO - TABLA N..$^{\circ}$ XVII (1514)}

\begin{tabular}{|c|c|c|c|c|c|c|c|c|c|c|}
\hline \multicolumn{3}{|c|}{ COMPRADOR } & \multicolumn{3}{|c|}{ VENDEDOR } & \multirow[b]{2}{*}{ Precio } & \multirow[b]{2}{*}{ Cantided } & \multirow[b]{2}{*}{ Especie } & \multirow[b]{2}{*}{ Plaszo } & \multirow[b]{2}{*}{ PROTOCOLO } \\
\hline Tirular & Oficio & Vecino & Titular & Oficio & Vecino & & & & & \\
\hline Luis Sánchez & & Murcia & Luis Madrid & mercader & Valencia & $5.700 \mathrm{mrs}$ & $1 \mathrm{mula}$ & & 1 anto & $362-713 r^{\circ}, v^{\circ}$ \\
\hline Francisco Martínez & & Murcia & Luis Madrid & mercader & Valencia & $3.187 \mathrm{mrs}$. & 1 mula & & $\begin{array}{l}\text { Todos los } \\
\text { Santos }\end{array}$ & $362.770 r^{\circ}, v^{\circ}$ \\
\hline Cristóbal Molina & sastre & Murcia & Luis Madrid & mercader & Valencia & $7.500 \mathrm{mrs}$ & 1 mula & & Santa M.: & $362-711 r^{\circ}, v^{\circ}$ \\
\hline Sebastián Garcja & & Murcia & Luis Madrid & mercader & Valencia & $2.625 \mathrm{mrs}$ & 1 rocín & & S. Miguel & $362.712 r^{\circ}, v^{\circ}$ \\
\hline Juan Castillo & & Murcia & Luis Madrid & mercader & Valencia & $3.000 \mathrm{mrs}$. & 1 rocin & & S. Juan & $362-714 \mathrm{r}^{\circ}, \mathrm{v}^{\circ}$ \\
\hline Alonso Vidal & & Murcia & Luis Madrid & mercader & Valencia & $7.500 \mathrm{mrs}$ & 1 mula & & Santa M. & $362-716 r^{\circ}, v^{\circ}$ \\
\hline Martin Gómez & & Murcia & Luis Mọreno & mercader & Murcia & $1.600 \mathrm{mrs}$ & 1 burra & & Santa M. & $362-718 r^{\circ}, v^{\circ}$ \\
\hline Pedro Martínez & & Murcia & Miguel Sevillano & & Murcia & $1.200 \mathrm{mrs}$ & 1 borrico & & mayo & $362-706 r^{\circ}, v^{\circ}$ \\
\hline Alonso Tahona & & Iniesta & Alonso Tarazona & & Belmonte & $1.875 \mathrm{mrs}$ & 1 asno & & S. Juan & $288-13 r^{*}, v^{*}$ \\
\hline
\end{tabular}


COMERCIO DE SEDA ELABORADA Y EN BRUTO - TABLA N. XVIII (1506-1515)

\begin{tabular}{|c|c|c|c|c|c|c|c|c|c|c|}
\hline \multicolumn{3}{|c|}{ COMPRADOR } & \multicolumn{3}{|c|}{ VENDEDOR } & \multirow[b]{2}{*}{ Precio } & \multirow[b]{2}{*}{ Cantidad } & \multirow[b]{2}{*}{ Especit } & \multirow[b]{2}{*}{ Plaseo } & \multirow[b]{2}{*}{ PROTOCOLO } \\
\hline Titular & Oficio & Vecino & Titular & Oficio & Vesino & & & & & \\
\hline Alonso Sta. Cruz & & Murcia & Ginds de Cartag & mercader & Murcia & $400 \mathrm{mrs}$. & 1 libra & & S. Migucl & $362-312 r^{\circ}, v^{\circ}$ \\
\hline Ginés Leơn & . & Murcia & Juancho Onate & genoves & Murcia & 910 mrs. & & & & $362-314 r^{*}, v^{*}$ \\
\hline Sancho Martince & sacristán & Molina & Diego Bacza & sastre & Murcia & $\begin{array}{l}2 \text { Duce- } \\
\text { dos } 1 / 2\end{array}$ & 2 libras $1 / 2$ & seda fina & Pascua & $362-545 r^{*}, v^{*}$ \\
\hline Alonso de Loón & & Murcia & Juan Perellón & 2 varas & Murcia & $500 \mathrm{mrs}$ & 2 varas & seda rasa negra & S. Juan & $608-176 v^{\circ}, t^{\circ}$ \\
\hline Diego de Contreras & & Murcia & Francisco Ruiz & mereader & Murcia & 13 reales & 1 vara $1 / 4$ & seda rasa negra & Reyes & $608-214 v^{*}, 215 r^{\circ}$ \\
\hline Pedro Ruiz & mercader & Murcia & Pedro Ruiz & corredor & Murcia & $800 \mathrm{mrs}$. & 2 libras & seda buena & Pascua & $603-35 r^{*}, v^{*}$ \\
\hline & barbero & Murcia & Luis Arroniz & mercader & Murcia & $562 \mathrm{mrs}$ & 2 libras & seda hilada & Pascuna & $288 \cdot 2 r^{*}, v^{*}$ \\
\hline$\longrightarrow$ & & & Luis Arroniz & mercader & Murcia & & 2 libras & seda hilada & Pascua & $288-3 r^{\circ}, v^{*}$ \\
\hline
\end{tabular}


COMERCIO DE HOJA DE MORERA - TABLA N. XIX (1506-1514)

\begin{tabular}{|c|c|c|c|c|c|c|c|c|c|c|}
\hline \multicolumn{3}{|c|}{ COMPRADOR } & \multicolumn{3}{|c|}{ VENDEDOR } & \multirow[b]{2}{*}{ Precio } & \multirow[b]{2}{*}{ Cantidad } & \multirow[b]{2}{*}{ Especie } & \multirow[b]{2}{*}{ Plaze } & \multirow[b]{2}{*}{ PROTOCOLO } \\
\hline Titular & Oficio & Vecino & Titular & Oficio & Verino & & & & & \\
\hline Ginés Pascual & alpargatero & Murcia & Francisco Fuster & & Murcia & $\begin{array}{l}40 \text { reales } \\
\text { castellanos }\end{array}$ & 21 moreras & & S. Juan & $608-100 r^{\circ}, v^{\circ}$ \\
\hline Ginés de Cartag & a mercader & Murcia & Martín Cánovas & & Murcia & $\begin{array}{l}13 \text { reales } \\
\text { castellanos }\end{array}$ & 7 moreras & & & $362-52 v^{*}, r^{*}$ \\
\hline Catalina Oller & viuda & & Francisca Soto & & Murcia & $2300 \mathrm{mrs}$ & 24 moreras & & S. Juan & $362-64 r^{*}, v^{*}$ \\
\hline Diego López & & Puebla & Salvador Navarso & mercader & Murcia & $542 \mathrm{mrs}$ & 7 moreras & & S. Juan & $603-21 r^{*}, v^{*}$ \\
\hline Pedro Valero & clérigo & Murcin & Antonio Moreno & clérigo & Murcia & $1329 \mathrm{mrs}$. & & & Pascua & $362-453 r^{*}, v^{*}$ \\
\hline
\end{tabular}


COMERCIO DE TRIGO Y CEBADA - TABLA N. XX (1506)

\begin{tabular}{|c|c|c|c|c|c|c|c|c|}
\hline COMPRADOI & & VENDEDOR & & & & & & \\
\hline Tifular & Verino & Titular & Vecino & Precio & Cantidad & Especie & Plase & PROTOCOLO \\
\hline Fernando Baeza & Baza & Alonso Agente & Murcia & Pragmática & $\begin{array}{l}2 \text { cahices } \\
1 \text { fanega }\end{array}$ & trigo & S. Juan & $608-93 r^{\circ}, v^{*}$ \\
\hline Femando Bacea & Bzza & Alvaro Aledo & Murciz & Pragmátia & 2 cahices & trigo & S. Juan & $608-93,94$ \\
\hline Guzmin & Mlurcia & Balibrea & Murcia & $410 \mathrm{mrs}$ & 4 cahices & trigo & S.Juan & $608-98 t^{*}, v^{\circ}$ \\
\hline Fernando Bacza & Baza & Alonso Lopez & Murcia & Pragmática & 2 cahices & trigo & S. Juan & $608-110 r^{\circ}, v^{\circ}$ \\
\hline Femando Bacza & Baza & Lorenzo de Relaza & Murcia & Pragmática & 1 cahiz & trigo & S. Juan & $608-120 r^{\circ}, v^{*}$ \\
\hline Fernando de Baeza & Baza & Juan Garcín & Murcia & Pragmática & 1 cahiz & trigo & S. Juan & $608-136 r^{\circ}, v^{*}$ \\
\hline Juan Murcia & Murcia & Juan de la Jara & Alcantarilla & a Pragmítica & $1 / 2$ cahiz & trigo & S. Juan & $608-142 v^{\circ}, 143 r^{\circ}$ \\
\hline Juan Murcia & Murcia & Juan de la jara & Alcantarilla & a Pragmática & $1 / 2$ cahiz & cebada & S. Juan & $608-142 x^{\circ}, 143 r^{\circ}$ \\
\hline Fernando Baeza & Bara & Juan Arroniz & Nora & Pragmática & 2 cahices & trigo & S. Juan & $608-141 v^{*}, 142 r^{\circ}$ \\
\hline Ginés de Cartagena mereader & Murcia & Pedro Quebral & Murcia & $400 \mathrm{mrs}$ & 1 cahiz & trigo & S. Juan & $362-533 r^{\circ}, v^{*}$ \\
\hline Alonso Chinchilla & Totana & Francisco Monteal & Muscia & & 1 cahiz & trigo & Carnestol. & $362-5478^{\circ}, v^{*}$ \\
\hline Juan Garcja & Murcia & Gines de Cartagena mercader & Murcia & $300 \mathrm{mrs}$. & & trigo & S. Juan & $362-553 r^{*}, v^{\bullet}$ \\
\hline Ma Aranda & Murcia & Ginés de Cartagena mercader & Murcia & $1.157 \mathrm{mrs}$. & 6 fanegas & trigo & S.Juan & $362-560 r^{\circ}, v^{*}$ \\
\hline Francisco Riquelme & Puebla & Ginés de Cartagena mercader & Murcia & 480 mrs. & 2 fanegas & trigo & S. Juan & $362-568 r^{\circ}, v^{\circ}$ \\
\hline Garcla Prieto & Liétor & Francisco Castilla & Murcia & $1.500 \mathrm{mrs}$. & 5 fanegas & trigo & S. Juan & $608-207 r^{\circ}, v^{*}$ \\
\hline Pedro Cisneros & Murcia & Gines de Cartagena mereader & Murcia & $400 \mathrm{mrs}$. & 1 cahiz & trigo & S. Juan & $362-42 r^{\circ}, v^{*}$ \\
\hline Pedro Cisneros & Murci2 & Ginés de Cartagena mercader & Murcia & $200 \mathrm{mrs}$ & 6 fanegas & cebada & S. Juan & $362-42 r^{\circ}, v^{\circ}$ \\
\hline
\end{tabular}


COMERCIO DE TRIGO Y CEBADA - TABLA N. XXI (1510)

\begin{tabular}{|c|c|c|c|c|c|c|c|c|c|}
\hline \multicolumn{2}{|c|}{ COMPRADOR } & \multicolumn{3}{|c|}{ VENDEDOR } & \multirow[b]{2}{*}{ Precio } & \multirow[b]{2}{*}{ Cantidad } & \multirow[b]{2}{*}{ Especie } & \multirow[b]{2}{*}{ Plaso } & \multirow[b]{2}{*}{ PROTOCOLO } \\
\hline Tibular & Vecino & Titular & Oficio & Vecino & & & & & \\
\hline Pefialvar & Puebla & Salvador Navarro & mercader & Murcia & $600 \mathrm{mrs}$ & 1 cahiz & trigo & S. Juan & $603-2 r^{*}, v^{*}$ \\
\hline Francisco Tomis & Puebla & Salvador Navarro & mercader & Murcia & $600 \mathrm{mrs}$ & 1 cahiz & trigo & S. Juan & $603-3 r^{*}, v^{*}$ \\
\hline Juan Autión & Puebla & Salvador Navarro & mercader & Murcia & $1.200 \mathrm{mrs}$. & 2 cahices & trigo & S. Juan & $603-4 r^{\circ}, v^{\circ}$ \\
\hline Pedro Garcla & Murcia & Salvador Navarro & mercader & Murcia & $900 \mathrm{mrs}$ & $1 / 2$ cahiz & trigo & S. Juan & $603-6 r^{\circ}, v^{*}$ \\
\hline Isabel Cemella & Murcia & Salvador Navarro & mercader & Murcia & $600 \mathrm{mrs}$ & 1 cahiz & trigo & S. Juan & $603-7 r^{*}, v^{*}$ \\
\hline Diego Contreras & Murcia & Pedro Ruiz & mercader & Murcia & $750 \mathrm{mrs}$. & 5 fanegas & trigo & S. Juan & $603-9 r^{\circ}, v^{\circ}$ \\
\hline Juan Auñón & Puebla & Salvador Navarro & mercader & Murcia & $550 \mathrm{mrs}$ & 1 cahiz & trigo & s. Juan & $603-15 r^{\circ}, v^{\circ}$ \\
\hline Fernando Albornoz & Puebla & Salvador Navarro & mercader & Murcia & 550 mrs. & 1 cahiz & trigo & S. Juan & $603-18 r^{\circ}, v^{\circ}$ \\
\hline Francisco Tomas & Puebla & Salvador Navarto & mercader & Murcja & $640 \mathrm{mrs}$. & 9 barchillas & trigo & S. Juan & $603-19 r^{\circ}, v^{\circ}$ \\
\hline Juan Bezón & Puebla & Salvador Navarro & mercader & Murcia & $550 \mathrm{mrs}$ & 1 cahiz & trigo & S. Juan & $603-20 r^{\circ}, v^{\circ}$ \\
\hline Diego Zambrana & Puebla & Salvador Navarro & mercader & Murcia & $550 \mathrm{mrs}$. & 1 cahiz & trigo & S. Juan & $603-22 r^{\circ}, v^{\circ}$ \\
\hline Alonso Tejada & Puebla & Salvador Navarro & mercader & Murcia & $275 \mathrm{mrs}$ & 1 cahiz & trigo & S. Juan & $603-22 v^{\circ}$ \\
\hline Juana Manuel & Puebla & Salvador Navarro & mercader & Murcia & $550 \mathrm{mrs}$. & 1 cahiz & trigo & S. Juan & $603-23 r^{\circ}, v^{\bullet}$ \\
\hline Martín Gil & Pucbla & Salvador Navarro & mercader & Murcia & $550 \mathrm{mrs}$. & 1 cahiz & trigo & S. Juan & $603-24 r^{\circ}, v^{\circ}$ \\
\hline Fernando Garcia & Puebla & Salvador Navarro & mercader & Murcia & 275 mrs. & $1 / 2$ cahiz & trigo & S. Juan & $603-25 r^{\circ}, v^{\circ}$ \\
\hline Femando Garcia & Puebla & Salvador Navarro & mercader & Murcia & $550 \mathrm{mrs}$. & 1 cahiz & trigo & S. Juan & $603-27 \mathrm{r}^{\circ}, \mathrm{v}^{\circ}$ \\
\hline
\end{tabular}


COMERCIO DE TRIGO Y CEBADA - TABLA N. XXII (1510-1514)

\begin{tabular}{|c|c|c|c|c|c|c|c|c|c|c|}
\hline \multicolumn{3}{|c|}{ COMPRADOR } & \multicolumn{3}{|c|}{ VENDEDOR } & \multirow[b]{2}{*}{ Precio } & \multirow[b]{2}{*}{ Cantidad } & \multirow[b]{2}{*}{ Especie } & \multirow[b]{2}{*}{$P / \infty \infty \infty$} & \multirow[b]{2}{*}{ PROTOCOLO } \\
\hline Titular & Ofirio & Verino & Titular & Ofrio & Vecinto & & & & & \\
\hline Juan Botclano & & Murcia & Pedro Ruiz & mercader & Murcia & $550 \mathrm{mrs}$. & 1 cahiz & trigo & S. Juan & $603-28 r^{*}, v^{*}$ \\
\hline Junn Garcia & & Murcia & Salvaxlor Navnsto & mercader & Murcia & 275 mrs. & 1/2 cahiz & trigo & S. Juin & $603-29 r^{*}, v^{*}$ \\
\hline Pedro Real & & Puebla & Salvador Navarro & mercader & Mureia & $250 \mathrm{mrs}$ & 1/2 cahiz & cebada & S. Juan & $603-30 r^{*}, v^{*}$ \\
\hline Alonso Tcjada & & Puebla & Salvador Navarro & mercader & Murcin & $275 \mathrm{mrs}$ & 1/2 cahiz & trigo & S. Juan & 603-31r, $v^{*}$ \\
\hline Juan Scgura & & Murcia & Pedro Ruiz & mercader & Murcia & $550 \mathrm{mrs}$. & 1 cahiz & trigo & S. Juan & $603-34 r^{*}, v^{*}$ \\
\hline Dicgo Zambrana & & Puebla & Salvador Navarro & mercader & Murcia & $500 \mathrm{mrs}$. & 1 eahiz: & 'trigo & S. Junn & $603-43 r^{*}, v^{*}$ \\
\hline Diego Sarramad & & Murcia & Salvador Navarro & mercader & Mureia & $500 \mathrm{mrs}$ & 1 cahiz & trigo & S. Juan & $603-42 r^{*}, v^{*}$ \\
\hline Lorenzo Setrano & & Murcia & Salvador Navarro & mercader & Murcia & 450 mrs. & & trigo & $\begin{array}{l}\text { Santa MS: } \\
\text { agosto }\end{array}$ & $603-42 r^{*}, v^{*}$ \\
\hline $\begin{array}{l}\text { Felipe liscaja } \\
\text { Juna Rey }\end{array}$ & genoveses & Murcia & Juan Cabrero & $\begin{array}{l}\text { comen- } \\
\text { dador }\end{array}$ & Murcin & $\begin{array}{l}126.000 \\
\text { mars. }\end{array}$ & 2.100 fancgas & cebacla & Pascua & $603-54 r^{*}, v^{*}$ \\
\hline Juan Garcla & & Murcia & Salvacior Navarro & mercader & Murcin & $3.408 \mathrm{mrs}$. & $\begin{array}{l}6 \text { cahices } \\
3 \text { varas pano }\end{array}$ & $\begin{array}{l}\text { trigo } \\
\text { dieciocl.. }\end{array}$ & S. Juan & $562-522 r^{*}, 523$ \\
\hline Juan Soto & & $\begin{array}{l}\text { lugar } \\
\text { Fajardo }\end{array}$ & Juan L.uján & & Alcantarilla & $450 \mathrm{mrs}$ & & yanizo & julio & $362-403 r^{*}, v^{\circ}$ \\
\hline Diego Abril & & Murcia & Martín Cabrero & $\begin{array}{l}\text { comen- } \\
\text { dador }\end{array}$ & Aledo & $940 \mathrm{mrs}$. & 1 eahiz & trigo cebada & julio & $362-409 r^{\circ}, v^{\circ}$ \\
\hline Diego Martínez & & Murcia & Salvador Navarso & mereader & Murcia & $200 \mathrm{mrs}$. & $1 / 2$ cahiz & panizo & julio & $362-452 r^{\circ}, v^{*}$ \\
\hline Diego Gómez & & Murcia & Pedro Aledo & mercader & Murcia & $650 \mathrm{mrs}$ & 1 cahiz & trigo & S. Juan & $362-484 r^{\circ}, v^{\circ}$ \\
\hline Alonso Doria & & Murcia & Dicgo Balibrea & mereader & Murcin & 1.400 mrs. & 1 cahiz & trigo & S. Juan & $362-487 r^{\circ}, v^{\circ}$ \\
\hline Diego Gómez & & Murcia & Diego Balibren & mercader & Murcia & $700 \mathrm{mrs}$. & 1 cahiz: & trigo & S. Juan & $362-489 r^{\circ}, r^{\circ}$ \\
\hline Pedro Noguera & & Murcia & Lorenzo Ferrer & mercader & Murcia & $2.200 \mathrm{mrs}$ & 4 cahices & trigo & abril & $362-723 r^{\circ}, v^{\circ}$ \\
\hline
\end{tabular}

